\title{
Distinction between Super-Cooled Water and Ice with High Duty Cycle Time- of-Flight Neutron Imaging
}

\author{
M. Siegwart ${ }^{1,2}$, R. Woracek ${ }^{3}$, J. I. Márquez Damián ${ }^{4}$, A. S. Tremsin ${ }^{5}$, V. Manzi-Orezzoli ${ }^{1}$, M. Strobl $^{2}$, \\ T. J. Schmidt ${ }^{1,6} \&$ P. Boillat ${ }^{1,2, *}$
}

${ }^{1}$ Electrochemistry Laboratory (LEC), Paul Scherrer Institut (PSI), 5232 Villigen PSI, Switzerland

${ }^{2}$ Laboratory for Neutron Scattering and Imaging (LNS), Paul Scherrer Institut (PSI), 5232 Villigen PSI, Switzerland

${ }^{3}$ European Spallation Source ESS ERIC, SE-221-00, Lund, Sweden

${ }^{4}$ Neutron Physics Department and Instituto Balseiro, Centro Atómico Bariloche, CNEA R8402AGP, Argentina

${ }^{5}$ University of California at Berkeley, Berkeley, CA 94720, United States

${ }^{6}$ Laboratory of Physical Chemistry, ETH Zürich, 8093 Zürich, Switzerland

\section{Abstract}

We report on measured neutron cross section data for super-cooled water and ice by time-of-flight neutron transmission imaging. In particular we demonstrate the use of high duty cycle time-of-flight (HDC-TOF) measurements to determine the local aggregate state of water with spatial resolution, by exploiting the neutron cross section dependence on the mobility of hydrogen atoms for long neutron wavelengths (> $4 \AA$ ) . While one can envision many different applications for this method, one example is to provide insights into the freezing mechanism during the start-up of polymer electrolyte fuel cells from below zero degrees. Unlike for other wavelength selective measurements (e.g. Bragg edge imaging), only a limited wavelength resolution is required for this method. With a chopper setup with high duty cycle (30\%), we reached a high contrast-to-noise ratio (CNR) with a contrast between ice and super-cooled water of $8 \%$. To maximize the CNR, we optimized the duty cycle, pulse period and image processing parameters. Moreover, we present a theoretical framework for performing such optimization calculations, which can be used to maximize CNR for any beam line and any substances. For the optimization procedure presented in this publication, we used cross section values for ice and super-cooled water measured with high wavelength resolution using wavelength frame multiplication choppers. Our results show that the aggregate state of water of a sufficiently thick layer of water (>0.5 $\mathrm{mm}$ ) can be reliably determined for a small area $\left(1 \mathrm{~mm}^{2}\right)$ and with a reasonable short acquisition time of 5 minutes. 


\section{Introduction}

Neutron transmission imaging (NTI) is a non-destructive method to study materials and processes occurring behind dense materials that are opaque to light and X-Rays. While light elements produce little contrast in X-ray transmission imaging, hydrogen strongly scatters neutrons. Therefore, NTI gained importance for the observation and quantification of hydrogen or water in various research sectors ${ }^{1}$. The range of applications for which quantification of water is crucial, spans from devices used in the renewable energy sector (e.g. fuel cells ${ }^{2-7}$ and electrolyzers ${ }^{8-10}$ ), over fluid flow in porous media ${ }^{11}$ and concrete processing ${ }^{12-14}$ to biological samples ${ }^{15-17}$. Among other topics, start-up of polymer electrolyte fuel cells (PEFCs) below zero degrees (cold start) was addressed by various studies in the past 12 years using NTI ${ }^{18-24}$. Because the water produced by the electrochemical reaction of the PEFC is ultra-pure, it can be present in its super-cooled state, which means it is liquid at temperatures below the freezing point ${ }^{18,25-27}$. The phase transition to ice is a stochastic process. The ice nucleation probability depends on the water volume, the surface characteristics of the material water is in contact with (e.g. container), cooling rate and final temperature as reviewed by Manson ${ }^{28}$. Lowering the temperature increases the probability that ice nucleation is initiated by an impurity or by nuclei present on solid surfaces in contact with water. Once freezing is initiated, it propagates to all locations connected with a continuous water network. Ice blocks the pathways of reactant gases to the electrodes and super-cooled water, which freezes during the cold start or during freeze-thaw cycles, can induce damage to fuel cell materials and components ${ }^{24,27,29,30}$. Apart of NTI, optical $25,26,31,32$ and $X$-ray ${ }^{26,33,34}$ imaging have also been used to investigate the role of super-cooled water during cold starts, though these methods require very specific cell designs. Using the fact that the cross section of liquid water and ice differs at low neutron energies (long wavelengths), an improved NTI method was demonstrated in our research group, to investigate the spatial distribution of super-cooled water and ice. The method employs a beryllium filter to shape the neutron beam spectrum by providing energy selectivity ${ }^{35,36}$. Using this method, Stahl et al. reported the evidence of local freezing events during a PEFC cold start ${ }^{37}$. The beryllium filter method made it possible to measure with a large neutron flux, but led to a low contrast between liquid water and ice (1.6 \%), which makes the method very sensitive to systematic measurement errors.

Here, we demonstrate that time-of-flight neutron transmission imaging with high duty cycle (HDCTOF) and hence with low wavelength resolution can be used to significantly increase the contrast, while keeping the neutron flux at a level that results in an acceptable contrast-to-noise ratio (CNR). The major difference to previously reported and routinely applied TOF-NTI approaches ${ }^{38}$ is that we take advantage of the fact that only a limited neutron wavelength resolution is required allowing for 
a high duty cycle (broad neutron pulses) providing a correspondingly high flux. The pulse parameters (e.g. overlap and wavelength resolution) and image processing parameters have to be optimized to maximize the CNR. We present in this work a theoretical framework for performing such optimization calculations, for which cross section values measured with high wavelength resolution are necessary. Experimentally measured cross section data for ice was reported by Torres et al..$^{39}$ at a temperature of $-158^{\circ} \mathrm{C}(115 \mathrm{~K})$, and was shown to be significantly smaller than that of liquid water at room temperature for long neutron wavelengths. To our knowledge, no cross section values for supercooled water have been published to-date and cross section values for ice at a temperature near the freezing point can only be found in reference ${ }^{40}$. In this work, we report the total neutron cross section of ice and super-cooled water at a temperature close to zero $\left(-2^{\circ} \mathrm{C}\right)$ for neutron wavelengths between 2- $9 \AA$ measured with high wavelength resolution using the wavelength frame multiplication choppers of the test beam line (V20) of the European Spallation Source (ESS) at the Helmholtz Zentrum Berlin $^{41}$. This data served as an input for the optimization procedure to maximize CNR when using HDC-TOF.

As HDC-TOF allows measuring neutron cross section of elements wavelength dependent, it can be used to distinguish between any states or elements, for which the cross sections are similar for short wavelengths but different for long wavelengths. The method could be used, for example, for battery research to distinguish between hydrogen and lithium. These two elements have similar cross section at short neutron wavelength, but the cross section of lithium increases steeper as a function of wavelength than the cross section of hydrogen. Further, the proton mobility and thus the neutron cross section not only depends on the aggregate state of water or aqueous solutions, but also on temperature ${ }^{42,43}$ and acidity. Therefore, HDC-TOF can be used to analyze the temporal and/or spatial evolution of $\mathrm{pH}$ or temperature (e.g. in fuel cells or electrolyzers). The theoretical framework to perform optimization calculations for maximizing CNR can be applied for any beam line and any substances, if cross section values, beam line wavelength spectrum and detector efficiency are available.

\section{Theoretical background}

The Beer-Lambert law describes transmission of neutrons through a sample:

$$
I=I_{o} e^{-\sigma_{T} \delta_{N} h_{S}}
$$

where $I_{0}$ is the intensity of the incoming neutron beam, $\sigma_{T}$ is the total neutron cross section, $\delta_{N}$ the atomic number density, $h_{S}$ is the thickness of the sample and $I$ is the intensity of the transmitted 
neutron beam. The number density is defined as the amount of atoms per volume and is therefore the product of the number of moles of atoms per volume (ratio between mass density and molar mass, $\delta_{m} / M$ ) and Avogadro constant. Commonly, the Beer-Lambert law is written using the linear attenuation coefficient $\Sigma=\sigma_{T} \delta_{N}$. Equation (1) is valid, when transmission images are corrected for scattered neutrons. When measuring water in a calibration container with known water thickness $h_{\mathrm{H}_{2} \mathrm{O}}$ the total cross section is calculated based on the ratio of the transmitted beam through the dry calibration container $I$ and container with water $I$

For simplicity, we refer hereafter to $I_{(\text {empty container })}$ as $I_{0}$, to $I_{\left(\text {empty container }+\mathrm{H}_{2} \mathrm{O}\right)}$ as $I$ and to $h_{\mathrm{H}_{2} \mathrm{O}}$ as $h_{S}$. The total cross section has the unit of an area $\left(10^{-24} \mathrm{~cm}^{2}=1\right.$ barn) and is a measure for the probability of interaction between a neutron and a nucleus, which hinders the transmission of the neutron in a straight trajectory through the material. Possible interactions are coherent or incoherent scattering and absorption. Scattering interactions are termed elastic if no energy transfer occurs between the neutron and nucleus, and inelastic in the opposite case. The total cross section is the sum of all partial cross sections:

$$
\sigma_{T}=\sigma_{\text {coherent, }, \text { lastic }}+\sigma_{\text {coherent, inelastic }}+\sigma_{\text {incoherent,elastic }}+\sigma_{\text {incoherent,inelastic }}+\sigma_{\text {absorption }}
$$

The kinetic energy of cold neutrons is of the same order of magnitude as the energy required for exciting translational oscillations in the water molecule related to diffusion. As diffusion decreases with temperature and is much slower in ice than in liquid water, the incoherent inelastic scattering cross section decreases with temperature and is lower for ice compared to super-cooled water. Therefore, the total cross section of water at long neutron wavelengths decreases with temperature and is substantially lower for frozen water compared to super-cooled water. For simplicity, we refer to the total cross section as cross section here after.

Most common applications of TOF-NTI are designed to detect sharp features that exist in the neutron transmission spectra of crystalline materials (i.e. Bragg-edge imaging) ${ }^{38}$. For this kind of application, wavelength resolutions $\leq 10 \%$ are generally necessary. TOF measurements with wavelength resolutions $\leq 3 \%$ are most efficient at state-of-the-art pulsed spallation sources where TOF-NTI is usually performed, but short pulses can also be produced by placing a rotating chopper disk with a narrow window into the beam. Measuring with short pulses of neutrons allows separating them by 
their velocity $v$ by knowing their arriving time at the detector $t_{T O F}$ and the distance between pulse source and detector $L_{d e t}$. The wavelength of the neutrons arriving at the detector can then be determined with equation (4) using the de Broglie relation, which relates the wavelength $\lambda$ of a particle with its momentum $p=m v$ and the Planck constant $h$ :

$$
\lambda=\frac{t_{T O F} h}{L_{d e t} m}
$$

where $m$ is the mass of the neutron.

When aiming for distinction between super-cooled water and ice during in situ studies, it is beneficial to maximize the flux on the sample and hence CNR. The present study therefore uses, in contrast to conventional TOF-setups, a chopper configuration with a comparably very large duty cycle that produces broad neutron pulses. Considering the neutron cross sections of super-cooled water and ice, we know that they are almost identical at short wavelengths, while super-cooled water attenuates neutrons stronger than ice at long wavelengths. To identify phase changes in a system with known water thicknesses in beam direction, one could simply acquire transmission images using only neutrons with long wavelengths to retrieve $\Sigma_{L W}$ :

$$
\Sigma_{L W}=\frac{-\ln \left(\frac{I_{L W}}{I_{0, L W}}\right)}{h_{S}}=\sigma_{T, L W} \delta_{N}
$$

However, in practical applications such as PEFCs, one does often not know the thickness nor the aggregate state of the water. For example, during cold starts of PEFCs super-cooled water and/or ice is produced over time. Therefore, a reference measurement of the attenuation at short neutron wavelengths $\Sigma_{S W}$ is necessary:

$$
\Sigma_{S W}=\frac{-\ln \left(\frac{I_{S W}}{I_{0, S W}}\right)}{h_{S}}=\sigma_{T, S W} \delta_{N}
$$

We can then determine the relative attenuation coefficient $\alpha_{r e l}$, which is the ratio between $\Sigma_{L W}$ and $\Sigma_{S W}$, and independent of the water thickness and density: 


$$
\alpha_{r e l}=\frac{\Sigma_{L W}}{\Sigma_{S W}}=\frac{\sigma_{T, L W}}{\sigma_{T, S W}}
$$

Based on the relative attenuation coefficients of super-cooled water $\alpha_{s c}$ and ice $\alpha_{i c e}$ the contrast is calculated using equation (8):

$$
\text { contrast }=\left(\frac{\alpha_{s c}-\alpha_{i c e}}{\alpha_{s c}}\right)
$$

For a reliable distinction between super-cooled water and ice, we want to reach a high contrast and low noise. The main source for noise in neutron transmission imaging is shot noise, which originates from the Poisson distribution of a particle beam hitting a detector. In case the average amount of neutron counts at the detector $I$ per evaluated area is sufficiently large (> 200), the Poisson distribution can be approximated with a Gaussian distribution with mean $I$ and relative standard deviation $\frac{\sqrt{I}}{I}=\frac{1}{\sqrt{I}} . I$ is proportional to the neutron flux, the pixel size (or evaluation area in case binning is applied) and the image acquisition time. The relative standard deviation decreases with longer acquisition times or increasing evaluation area when the neutron flux is constant. To distinguish between super-cooled water and ice during cold starts of PEFCs, the desired image acquisition time is less than a few minutes and spatial resolution has to be high enough to resolve water/ice in small regions of interest. To assess whether a technique is useful for practical applications involving dynamic processes in which time is a relevant factor, the CNR must be considered.

To obtain optimal contrast between the relative attenuation coefficients of the two aggregation states and at the same time keeping the flux as high as possible to reduce shot noise, the experimental setup as well as imaging processing parameters have to be chosen carefully. These parameters are namely the duty cycle of the chopper disk, the pulse period, which is controlled by the rotation frequency of the chopper disk, and the time intervals for short and long neutron wavelengths selected during image processing. To obtain the highest possible CNR with a given image acquisition time and evaluation area, an optimization procedure can be used to find the optimal combination of chopper disk parameters (i.e. duty cycle and pulse period) and image processing parameters (boundaries for short and long wavelengths, respectively). This optimization procedure is useful to prepare experiments at any beam line and requires as inputs only the wavelength spectrum of the beam line, detector efficiency as a function of wavelength and reference cross sections of the materials of interest with a high wavelength resolution. The theoretical framework for such optimization calculations is described 
in Appendix $A$ and the results are presented in the Results \& Discussion section in the subsection "Optimization of CNR".

\section{Experimental}

Water wedge calibration container

All herein presented experiments were performed using a water wedge calibration container machined from aluminum with four sections corresponding to different water/ice thicknesses between the body (Figure 1a) and the cover (Figure 1b). The thicknesses were 0.133, 0.261, 0.515 and $1.011 \mathrm{~mm}$ (Table 1$)$. The smallest thickness $(0.133 \mathrm{~mm})$ is within the order of magnitude of the water thickness present in a gas diffusion layer and the largest two thicknesses (0.515 and $1.011 \mathrm{~mm})$ are within the order of magnitude of the channel depth of flow fields used for fuel cells. Due to fabrication inaccuracies, the space between body and cover (measured using a Keyence Wide-Area 3D Measurement System) varies over the section areas (see Table 1).

The calibration container was filled with ultrapure water (Milli-Q) through a channel behind the four holes at the bottom, while the holes on top were connected through a channel to an outlet pipe and allowed air to escape. Water in the four sections was connected through cavities with $1 \mathrm{~mm}$ depth (see Figure 1a). For drying, pressurized air was first connected to the top channel and later to the bottom channel. The calibration container was not filled completely with water in order to leave room for volume expansion during the phase transition to ice. The total sealed area inside the O-ring was $3 \times 3 \mathrm{~cm}^{2}$ and slightly larger than the field of view of the detector. Borated aluminum plates with a width of $3 \mathrm{~mm}$ and thickness of $5 \mathrm{~mm}$ in beam direction were placed at the backside of the calibration container. Borated aluminum absorbs neutrons (compare Figure 2) and the neutron counts measured in the area behind it were used to correct transmission images for scattered neutrons.

\section{Climate chamber and temperature cycle}

The closed calibration container was placed into an aluminum box, which was continuously flushed with pressurized air to avoid condensation of water at the surface of the calibration container and Peltier elements were used to regulate its temperature. After filling the container with water it was cooled down to a steady temperature of $6{ }^{\circ} \mathrm{C}$ and images of liquid water were acquired. Then, the temperature was decreased to $-2{ }^{\circ} \mathrm{C}$ in order to acquire images of super-cooled water (no freezing occurred). Ultrapure water (Milli-Q) inside an aluminum calibration container stays in super-cooled state even at temperatures of a few degrees below $0{ }^{\circ} \mathrm{C}$ (see Introduction). To trigger the phase 
transition from super-cooled water to ice the calibration container was cooled down to $-13{ }^{\circ} \mathrm{C}$ and then the temperature was increased slowly to $-2^{\circ} \mathrm{C}$, where images of ice were acquired.

\section{Neutron imaging}

The experiments were performed at the test beam line (V20) of the ESS, which is located at the BER II reactor at the Helmholtz Zentrum Berlin ${ }^{41,44}$. We used a neutron counting detector with Micro Channel Plates (MCPs) and Timepix readout with $512 \times 512$ pixels, $55 \mu \mathrm{m}$ pixel pitch and a field of view of $2.8 \times 2.8 \mathrm{~cm}^{2}$, allowing to record many time bins per pulse period ${ }^{45}$. Neutron counts in each pixel and time bin were summed over many TOF cycles, and each acquisition cycle was triggered by a TTL signal with the same frequency as the source pulse choppers. The total exposure time for an image of one time bin is termed acquisition time. Measurement were performed in a high (i.e. with WFM choppers) and a low (i.e. with HDC) wavelength resolution mode using the flexible chopper cascade of V20.

Wavelength frame multiplication (WFM) (high wavelength resolution): These measurements were needed to determine the neutron cross sections of ice and super-cooled water with high wavelength resolution and they were carried out using the ESS source pulse choppers (mimicking the long pulsed future ESS source) together with the WFM choppers. The WFM concept will also be utilized at several ESS instruments, for example at the future imaging instrument $\mathrm{ODIN}^{46}$ and a detailed explanation can be found in references ${ }^{41,44}$. For the measurement presented in this paper, the WFM system was set up to yield a constant wavelength resolution of $2 \%$ across the spectrum, which was achieved by setting the distance between the optically blind WFM pulse shaping chopper disks to $427 \mathrm{~mm}^{44}$. The acquisition time per image stack was $1 \mathrm{~h}$, with a stack consisting of 1014 images and each image corresponding to an individual time bin within a chopper repetition period ( ). Five image stacks were recorded for each state (open beam, dry reference image, ice, super-cooled water and water at $6^{\circ} \mathrm{C}$ ) in order to obtain good statistics.

High duty cycle (HDC) (low wavelength resolution): The low wavelength resolution and high flux measurements were performed with a high duty cycle of the chopper disk system. For this purpose, the ESS source and WFM choppers were parked fully open and a duty cycle of $30 \%$ was achieved by setting the corresponding phase shift between the bandwidth choppers 1 and 2 of V20 (i.e. now acting as source pulse choppers $)^{44}$. The frequency of the chopper discs was set to $21 \mathrm{~Hz}$. With these settings, the burst time of the pulse was $14.3 \mathrm{~ms}$ and the period between pulses was $47.6 \mathrm{~ms}$ ( ). With a distance from the chopper discs to the detector of $16.3 \mathrm{~m}$ (flightpath), a pulse overlap of $7 \mathrm{~ms}$ was produced. 


\section{Image processing}

All images acquired with the MCP detector were corrected for the deficiency of the detector, which can only register timing of one neutron per image acquisition frame. This procedure is called event overlap correction ${ }^{47}$. The five image stacks per state (open beam, dry, ice, super-cooled, water) acquired with the WFM choppers were summed to one image stack per state to obtain higher statistics and the summed images were then "stitched" using Mantid to obtain a continuous spectrum from the six individual time frames recorded in a WFM measurement.

All images recorded in WFM and HDC experiments underwent the following processing:

1. Spatial $4 \times 4$ binning to increase neutron statistics.

2. Intensity threshold application for WFM data: For low intensity images small errors in the scattered background correction result in large errors in calculated cross section values. The images at individual time bins with neutron counts below a certain threshold value were not taken into account for further data analysis.

3. Time binning: Neutron statistics was increased by applying a time binning. For the treatment of the WFM data, the frames were summed in groups of 21. One time bin of a summed image corresponds to $1.43 \mathrm{~ms}$ and $0.29 \AA$, respectively. For the HDC data all frames within the short and long wavelength time windows were summed. The short wavelength window corresponds to all images with arrival times at the detector between $9-21 \mathrm{~ms}$ and the long wavelength window corresponds to 38 - $54 \mathrm{~ms}$, respectively. The neutron intensity weighted average wavelengths (Appendix A) for these time boundaries are 1.8 - 3.2 Å for the short and $7.1-9.4$ A for the long wavelength window.

4. Open beam normalization provides transmission $\left(1 / I_{0}\right)$ images independent of spatial beam and detector inhomogeneities through division of sample images by open beam image (image without sample).

5. Correction for scattered neutrons: All transmission images were corrected for scattered neutrons by evaluating the intensity behind materials opaque to neutrons (black bodies). For further details concerning this method see references ${ }^{48,49}$. The intensity of scattered neutrons behind borated aluminum was measured in three different regions of the field of view (Figure 2) for all transmission images (sample images divided by open beam). The scattered background intensity was then inter- and extrapolated in x-direction using a polynomial fit to the regions of interests where water/ice was present (black rectangles in Figure 2).

6. Intensity normalization: Intensity of neutron reactor output and detector efficiency fluctuate over time. To correct for these fluctuations, we calculated the ratio of the mean intensity in 
areas without water/ice (blue rectangles in Figure 2) between water/ice and dry reference images to obtain an intensity normalization correction factor. The water/ice images were then multiplied with this correction factor.

7. Referencing by dry image: Division of water/ice images by dry images (to obtain ratio between $I$ and $I$ as described in equation (2)).

8. Calculation of cross section for WFM measurements was performed with equation (2).

9. Calculation of mean relative attenuation and standard deviation for the HDC measurements: The mean relative attenuation coefficients for the two aggregate states (equation (7)) and their standard deviations were calculated based on 30 squares of $1 \mathrm{~mm}^{2}$ area per section (Figure $2 \mathrm{~b}$ ). This procedure allows to estimate the confidence level for the distinction between ice and liquid water with spatial resolution. In our experiments, the same aggregate state was present in the whole calibration container, but for other experiments, HDC-TOF should enable the analysis of the aggregates state with high spatial resolution.

\section{Results \& Discussion}

\section{Cross section of water and ice measured with WFM chopper system}

To obtain the cross section values of ice and super-cooled water at $-2{ }^{\circ} \mathrm{C}$ and water at $6{ }^{\circ} \mathrm{C}$ with high wavelength resolution, TOF-NTI measurements were performed with the WFM chopper system.

\section{Radiograms}

The radiograms in Figure 3 show that the experiment delivered successfully the desired aggregate states of water. In the image shown in Figure 3a, water is present in the calibration container in its super-cooled state at $-2{ }^{\circ} \mathrm{C}$. The presence of ice at $-2{ }^{\circ} \mathrm{C}$ after the triggering of freezing at lower temperature can be easily confirmed from the volume expansion visible in the transmission image (TI) of ice (highlighted with the red rectangle in section 4 in Figure 3b, where it is most pronounced). The volume expansion is less visible for sections $1-3$. The water thickness is smaller in these areas and the sections are adjacent to cavities of $1 \mathrm{~mm}$ depth (see Figure 1a). A volume expansion of water into the cavity on top of the sections results in a smaller height increase and volume expansion of water between two adjacent sections is not visible in the radiograms because these areas are behind the borated aluminum plates. 


\section{Cross section measurement of liquid water and ice}

Figure 4 shows the cross sections of super-cooled water and ice at a temperature of $-2{ }^{\circ} \mathrm{C}$. For both aggregate states, the cross section increases monotonously with longer neutron wavelength and does not show special features at specific wavelengths (i.e. Bragg edges). Since ordinary ice has a hexagonal crystal structure, one might expect to observe Bragg edges, but none can be observed in Figure 4. This can be explained by the fact that the incoherent scattering cross section for hydrogen is 46 times larger than the coherent scattering cross section ${ }^{50}$. The cross section values calculated (with equation (2)) for the mean thickness of $1.011 \pm 0.01 \mathrm{~mm}$ are shown with dots. The error bands show the uncertainties related to the thickness of water/ice $(1.011 \pm 0.01 \mathrm{~mm})$ only (darker error band) and including uncertainties due to the correction for scattered background (lighter error band), which contribute in the following way to errors in the cross section evaluation:

1. An underestimated thickness results in overestimated cross section values and vice versa.

2. If the neutron counts per pixel in the water/ice and dry reference images are not corrected for scattered neutrons, transmission of neutrons through water/ice is over- and cross section underestimated. The reverse is also true; an overestimation of scattered neutron intensity results in an underestimation of transmission and therefore overestimation of cross section values.

The intensity of scattered neutrons was measured in three areas of the field of view (Figure 2) and amounts on average to $2 \%$ of the open beam intensity. The extrapolation to the region of interest (section 4 in Figure 2) yields some uncertainty. To estimate the uncertainty band of the cross section values presented in Figure 4, we used a band of $1.00 \mathrm{~mm}$ to $1.02 \mathrm{~mm}$ for the water thickness and a band of $1 \%$ to $3 \%$ for the scattered background intensity. As the cover was screwed tight to the body of the water calibration container, the thickness of water/ice was constant during the whole experiment. Similarly, the uncertainty originating from the scattered background intensity extrapolation is almost independent of the phase of water present in the water calibration container. Therefore, while the absolute cross section values are afflicted with an uncertainty of about 10 barns at $8.7 \AA$ (5\% of absolute cross section value), the difference of the cross section measured for ice and super-cooled water is expected to suffer from much less uncertainty.

\section{Comparison of experimental data to physical model output}

A physical model implemented using the open source nuclear data processing program NJOY ${ }^{51}$ was used to compute the effect of temperature and aggregate state on the total neutron cross section per $\mathrm{H}_{2} \mathrm{O}$ molecule based on molecular dynamics simulations ${ }^{52,53}$. The vibrational spectra obtained from 
the simulations is used to compute the double differential cross section of hydrogen bound in water, which is later integrated to obtain the total scattering cross section. The comparison between the results of the model and the measurements performed with WFM choppers for super-cooled water and ice at $-2{ }^{\circ} \mathrm{C}$ and water at $6{ }^{\circ} \mathrm{C}$ is depicted in Figure 5 a, along with published reference cross section data $42,54,55$ for water at room temperature $\left(18-23^{\circ} \mathrm{C}\right)$ (Figure $\left.5 \mathrm{~b}\right)$. The measured data and the results of the model are in good agreement. Below $4 \AA$, where temperature has a smaller effect on the cross section of liquid water, the cross section values measured with WFM choppers are in good agreement with water cross section measured at room temperature and published in literature ${ }^{43,54,55}$. The following important trends can be observed for the measured as well as for the model data:

- At wavelength larger than $4 \AA$, the cross section of super-cooled water at $-2{ }^{\circ} \mathrm{C}$ increases steeper as function of wavelength compared to the one of ice, and water at $6{ }^{\circ} \mathrm{C}$ increases even steeper than super-cooled water at $-2{ }^{\circ} \mathrm{C}$. The reason for this behavior is an increase of inelastic scattering components in the cross section with higher mobility of protons.

- At the maximum wavelength probed in the experiment $(8.7 \AA)$, the effect of $8{ }^{\circ} \mathrm{C}$ temperature difference for liquid water (water at $6{ }^{\circ} \mathrm{C}$ compared to $-2{ }^{\circ} \mathrm{C}$ ) results in an increase of about 6 barns in cross section and ice has a cross section that is around 17 barns lower compared to super-cooled water at $-2{ }^{\circ} \mathrm{C}$.

- At wavelength lower than $3 \AA$, ice has a slightly higher cross section (up to 3 barns) compared to liquid water (shown with the insert in Figure 5a).

At long neutron wavelength, we measured ice and liquid water cross section values, which are lower compared to the model, but the deviation between measured cross section and the ones computed by the model is less than 10 barns and lies within the uncertainty range of the WFM cross section measurement as elaborated above. The experimental validation of the model is useful for any study where the neutron cross section of ice or liquid (super-cooled) water at different temperatures plays a role. Experimental measurements with high wavelength resolution can be time consuming and to obtain a reference cross section data set for different aggregates states or temperatures might be excessive in some cases. In contrast, the model can compute the cross section values for different aggregate states and temperatures over a wide wavelength range within a few minutes. Such data sets are useful to optimize neutron pulse and image processing parameters for HDC-TOF. For electrochemical applications such as fuel cells or electrolyzers, for example, the benefit of the validation of the model is obvious. As mentioned above, beside the interest of distinguishing between super-cooled water and ice during PEFC cold starts there are other applications for HDC-TOF. For 
example, visualizing a map of the water temperature inside such devices could help to improve cell components and designs in particular with regard to their durability. Also other water containing systems or in fact, any systems that contain elements where the neutron cross section varies with the wavelength spectrum can profit form HDC-TOF.

\section{Distinction of water and ice with HDC-TOF}

While the WFM measurements are useful to measure reference values for the cross sections, the distinction of liquid water from ice requires a much lower wavelength resolution. Measurements with two broad wavelength windows are sufficient to distinguish between super-cooled water and ice (equation (7)). Using the HDC setup, a much higher flux can be utilized, which allows to perform measurements within a few minutes.

\section{Relative attenuation coefficients, contrast and CNR as a function of water/ice thickness}

The measured average relative attenuation coefficients $\left(\alpha_{\text {rel }}\right)$ for ice and super-cooled water at $-2{ }^{\circ} \mathrm{C}$ as a function of thickness are shown in Figure 6. The error bars show the $99 \%$ confidence intervals. They are calculated based on the standard deviation $\left(\sigma_{\alpha_{r e l}}\right)$ of $\alpha_{\text {rel }}$ measured for 30 squares of $1 \mathrm{~mm}^{2}$ area per section (as shown in Figure 2 in the section "Experimental"). The time boundaries for the short wavelength window were $9-21 \mathrm{~ms}$ and for the long wavelength window $38-54 \mathrm{~ms}$, respectively. As the wavelength resolution is low ( $\Delta \lambda / \lambda=110 \%$ at $2.5 \AA$, Table 2$)$, we calculated the neutron intensity weighted average wavelength, (Appendix A) for these time boundaries, which are $1.8-3.2 \AA$ for the short and 7.1 - 9.4 ̊ for the long wavelength window (shown in Figure 9).

The relative attenuation coefficient is - as expected - lower for ice compared to super-cooled water. For the two larger thicknesses $(1.01 \mathrm{~mm}$ and $0.52 \mathrm{~mm})$, the $99 \%$ confidence intervals of the mean $\alpha_{\text {rel }}$ do not overlap. This indicates that a reliable distinction between super-cooled water and ice is possible for these thicknesses with an evaluation area of only $1 \mathrm{~mm}^{2}$.

Table 4 displays mean contrast, statistical noise $\left(\sigma_{\alpha_{r e l}}\right)$ and CNR for the four thicknesses of the same 30 areas per section as the results shown in Figure 6. The contrast between $\alpha_{\text {rel }}$ of super-cooled water and ice amounts to around $8 \%$. To test if a distinction with a given confidence level $(C$ ) is possible, one can calculate the margin of error and compare it with the CNR. If $C$ is chosen to be $99 \%$ (same as confidence interval in Figure 6), CNR has to be higher than 2 times the z-score for a two sided confidence interval of $99 \%$, which is 5.15 . The high noise level in our measurements for the lower 
thicknesses $(0.13$ and $0.26 \mathrm{~mm})$ do not allow for obtaining a suitable CNR. For the larger thicknesses (0.52 and $1.01 \mathrm{~mm}$ ) CNR is larger than 5.15 .

A contrast of $8 \%$ with a CNR larger than 5.15 for water/ice thicknesses of $0.52 \mathrm{~mm}$ and $1 \mathrm{~mm}$ is a promising result. It was obtained for an evaluation area of $1 \mathrm{~mm}^{2}$ and acquisition time of 5 minutes, with the neutron flux and spectrum of V20 (Table 2). For experiments performed with a beryllium filter at ICON (SINQ) the contrast was $1.6 \%^{36}$ and CNR was for an evaluation area of $5 \mathrm{~mm}^{2}$ and an acquisition time of 10 minutes in the same order of magnitude as the CNR reported here. However, the contrast is increased by a factor of five with HDC-TOF. This is an improvement, because a higher contrast is more robust against biases (e.g. scattered background correction). A good contrast and a reliable distinction for an evaluation area of $1 \mathrm{~mm}^{2}$ and acquisition time of 5 minutes confirms the potential of the method for the characterization of PEFC cold starts, where spatial and temporal resolution is important.

CNR increases as a function of water/ice thickness for the thicknesses probed in this experiment $(0.13-1 \mathrm{~mm})$. Because transmission of long wavelength neutrons through water/ice is low, there is an optimal water thickness at which the maximal CNR is reached, and CNR decreases for larger water thicknesses. For example, with the long wavelength window used in the HDC experiment (7.1 - 9.4 $\mathrm{A}$ ), transmission is already less than $10 \%$ for a water thickness of $3.5 \mathrm{~mm}$. The measurement of relative attenuation under conditions with low transmission is more sensitive to errors in scattered background correction and suffers from a higher noise level. To increase CNR for small water/ice thicknesses $(<0.5 \mathrm{~mm})$, one can increase the acquisition time and/or the evaluation area. Noise decreases proportional to the square root of the amount of neutron counts per evaluation area. Figure 7 shows that an evaluation area of $10 \mathrm{~mm}^{2}$ would be sufficient to measure a CNR above 5 for a water/ice thickness of $0.13 \mathrm{~mm}$.

\section{Optimization of CNR}

\section{Comparison of measured and predicted effective cross section}

Because of the broad source pulses, the neutrons arriving at a given time at the detector have a large spread of wavelengths (see Figure 8), and the cross section for each arrival time needs to be calculated as an effective cross section $\sigma_{\text {eff }}$ (Figure 8b), in the same manner as when calculating an effective cross section for white beam experiments. The effective cross section was calculated (see appendix A for details) for the middle pulse of a sequence of three pulses to take into account the pulse overlaps. 
Figure 9 shows the results obtained when calculating $\sigma_{e f f}$ for super-cooled water and ice as a function of time (bottom $x$-axis) and intensity weighted average wavelength (top $x$-axis) together with the experimentally measured $\sigma_{e f f}$. The calculations (see Appendix A) were performed for the experimental setup parameters given in Table 2 with the wavelength spectrum of V20 and cross section data measured using the WFM choppers. $\sigma_{e f f}$ measured with such a low wavelength resolution shows "artifacts" due to pulse overlap (first $7 \mathrm{~ms}$ of measurement) and wavelength resolution smearing effects. Nevertheless, it can clearly be seen from Figure 9 that, even with a low wavelength resolution and a few milliseconds of pulse overlap, a significant difference in cross section is measured at long neutron wavelength. In addition, Figure 9 shows a good agreement between the values for $\sigma_{\text {eff }}$ measured and calculated using the calculation sequence described in Appendix A.

\section{Influence of pulse and image processing parameters on CNR}

To find the maximum achievable CNR for the difference between $\alpha_{r e l}$ of water/ice, we used the calculation sequence described in Appendix $A$ and an optimization procedure, which was performed for different target contrast values. The following parameters were optimized: pulse period $t_{\text {period }}$, duty cycle (ratio between pulse burst time $t_{\text {puse }}$ and $t_{\text {period }}$ ) and boundaries for the short and long wavelength integration windows (image processing parameters). The distance between chopper and detector was fixed to be $16.3 \mathrm{~m}$ (Table 2), and we evaluated the maximum achievable CNR for an evaluation area of $1 \mathrm{~mm}^{2}, 5$ minutes acquisition time and a water/ice thickness of $0.1 \mathrm{~mm}$. The latter was chosen to facilitate noise estimation (see Appendix A).

Figure 10 visualizes the impact of pulse and image processing parameters on the resulting neutron flux and $\sigma_{e f f}$ of super-cooled water and ice measured over time. The target contrast values for the difference between $\alpha_{r e l}$ of ice and super-cooled water (equation (8)) were $2 \%, 6 \%$ and $11 \%$. The optimal pulse and image processing parameters to yield the maximal CNR and noise for these points are given in Table 5. A large duty cycle yields a high neutron flux (Figure 10a). The optimal wavelength windows are very broad and even overlapping in the time region where $\sigma_{e f f}$ for ice is equal to $\sigma_{\text {eff }}$ for super-cooled water. In this scenario, pulse overlap and wavelength resolution smearing are responsible for a small difference of the cross sections towards the end of the pulse. Consequently, a low contrast of only $2 \%$ is obtained. A smaller duty cycle results in larger contrast with the trade-off, that neutron flux is reduced, which leads to increased shot noise. A duty cycle of $20 \%$ (Figure 10c) yields comparably little pulse overlap and wavelength resolution smearing impacts $\sigma_{e f f}$ not significantly. Therefore, a good contrast (11 \%) between $\sigma_{e f f}$ for super-cooled water and ice is 
obtained. On the other hand, the high contrast is also reached due to comparably narrow wavelength integration windows and thus, neutron statistics is further reduced. The highest CNR is obtained with a duty cycle of $38 \%$ with medium wide wavelength integration windows, where the reduced contrast compared to a lower duty cycle is compensated with higher neutron statistics.

The results of the CNR maximization (pulse and image processing optimization) are plotted in Figure 11 as a function of contrast. The three points, which are highlighted in red, are the CNR obtained for the contrast values $2 \%, 6 \%$ and $11 \%$, for which $\sigma_{e f f}$ is plotted as a function of time in Figure 10 and results are given in Table 5. The maximal achievable CNR is high for contrasts between $4-8 \%$, which are reached with a chopper disk duty cycle between $20-50 \%$, when the disk rotation frequency and the boundaries for the wavelength windows are adjusted accordingly. At contrasts higher than $8 \%$ and lower than $4 \%$, CNR decreases rapidly. The maximal CNR is reached for a duty cycle of $38 \%$ and contrast of $6 \%$. However, when choosing a setup, it is important to note that the point with the highest CNR is not necessarily the best. If the "cost" in terms of CNR is moderate, using a higher contrast value will help to reject any systematic biases more effectively.

We measured the HDC-TOF data with a duty cycle of $30 \%$ and a pulse period of $47.6 \mathrm{~ms}$. These parameters are close to the parameters yielding the CNR highlighted in green in Figure 11. For the time boundaries reported above (short wavelength window $=9-21 \mathrm{~ms}$ and long wavelength window $=38-54 \mathrm{~ms}$ ), we calculate a CNR of 5.7 for a water/ice thickness of $0.1 \mathrm{~mm}$, with a contrast of $7.8 \%$. While a contrast of $7.7 \%$ was actually reached in the experiment, the obtained CNR of 1.8 is clearly lower than expected.

The latter discrepancy is due to the limitation on count rate of the neutron counting detector operating in high resolution timing (sub- $\mu \mathrm{s}$ ) mode, in which only one neutron can be detected per pixel within one acquisition frame. That deficiency of present Timepix-based detection technology led to degradation of detection efficiency for the very high incoming neutron flux, where many incoming neutrons were ignored by the pixels, which were busy processing previous events. As mentioned earlier, the accurate spectrum can still be recovered from the measured data ${ }^{56}$, but the lost neutron counts cannot be recovered and that leads to a lower measured CNR value compared to predictions. As demonstrated earlier in this paper, for accurate mapping of ice/liquid water distribution, the wavelength of neutrons does not have to be measured as accurately as in Bragg edge imaging. Therefore, we can operate in future experiments the existing MCP/Timepix detector in neutron counting mode, which limits the time resolution to the width of the acquisition shutter, but allows much higher neutron count input rates. Since neutrons within several wavelength bands can be combined in our analysis (as in equation (7) using only two bands) the CNR values close to the optimal 
are expected to be measured. In addition, next generation read-out electronics (e.g. Timepix3 or Timepix4) should allow for much higher input fluxes at high time (and thus wavelength) resolution mode. Beside this, other detector systems can be used for HDC-TOF. For example, cameras with gated light amplifiers are capable of acquiring images over defined time windows within a repeating cycle. Such cameras in combinations with neutron sensitive scintillators screens are useful for HDC-TOF, in case the optimal time windows for the two wavelength windows are known before the experiment. The optimal windows can be found, for example, with the theoretical framework described in this publication.

\section{Conclusion}

Time-of-flight neutron transmission imaging with high duty cycle (HDC-TOF) was demonstrated to be suitable for the spatially resolved distinction between ice and super-cooled water with good spatial and temporal resolution. The ratio between long and short wavelength attenuation $\alpha_{\text {rel }}$ was measured as a function of water/ice thickness. The contrast between $\alpha_{r e l}$ for the two aggregate states reaches $8 \%$ when optimal image acquisition and processing parameters are used. This is an improvement compared to using a neutron filter for providing energy-selectivity, which was shown to deliver a contrast of only $1.6 \%$. With an increased contrast, the measurement is less sensitive to biases. Using HDC-TOF with the neutron flux available at the beam line V20 at Helmholtz Zentrum Berlin, a measurement time of five minutes and an evaluation area of $1 \mathrm{~mm}^{2}$ is sufficient to measure a contrast-to-noise ratio (CNR) greater than 5 for water/ice thickness greater than $0.5 \mathrm{~mm}$. A reliable distinction for smaller water/ice thicknesses or a better temporal resolution for large water thicknesses is possible in case neutron statistics is increased. This can be achieved by increasing the detector efficiency using an improved counting mode of the detector, increasing the evaluation area (at the cost of lower spatial resolution), or by measuring at a neutron source with higher flux. On this basis, we can conclude that using HDC-TOF makes it possible to distinguish ice and super-cooled water with the temporal and spatial resolution needed to gain insights into the freezing mechanism during cold starts or freeze-thaw cycles of polymer electrolyte fuel cells.

In addition, water/ice cross sections were measured with high wavelength resolution and compared to simulations based on molecular dynamics. To our knowledge, this publication is the first report showing experimentally measured attenuation spectra for super-cooled water. The results show that at long neutron wavelengths ( $8.7 \AA$ ) the difference in cross section for ice and super-cooled water amounts up to 17 barns, while $8^{\circ} \mathrm{C}$ water temperature difference (between -2 and $6^{\circ} \mathrm{C}$ ) results in around 6 barns cross section difference. This is in good agreement with the simulations. 
Finally, a theoretical framework for pulse and image processing parameter optimization for the distinction between ice and liquid water was presented. The calculation sequence requires cross section values measured with high wavelength resolution (e.g. the experimentally measured cross section values or cross section data computed by the model presented in this publication). To estimate noise, we used a linearization approximation, which is justified for small water thicknesses. Therefore, the theoretical framework can be used to optimize pulse and image processing parameters for any beam line and any substances, when cross section values, beam line wavelength spectrum and detector efficiency are available and the thicknesses of the materials of interest are small. To optimize these parameters for larger material thicknesses, noise estimation should be adapted. Beside the distinction between liquid water and ice, HDC-TOF has potential for many other applications such as, for example, spatially and temporal resolved $\mathrm{pH}$ or water temperature measurements (e.g. for fuel cells or electrolyzers).

\section{Appendix A}

All variables introduced in this section are listed in Table 6. The effectively measured transmission of a polychromatic neutron beam is generally calculated by integrating the incoming and outgoing wavelength dependent intensity over the considered wavelength range:

$$
T_{\text {eff }}=\frac{\int_{\min }^{\lambda_{\max }} I_{0}(\lambda) \eta_{\mathrm{det}}(\lambda) e^{-\sigma_{T}(\lambda) \delta_{N} h_{s}} d \lambda}{\int_{\lambda_{\min }}^{\lambda_{\max }} I_{0}(\lambda) \eta_{\mathrm{det}}(\lambda) d \lambda} .
$$

For time-of-flight experiments with a broad source pulse, a similar integration has to be done, as the neutrons arriving at the detector at a defined measurement time $t_{\text {det }}$ cover a wide range of wavelengths (see Figure 8 and Figure 12). $t_{d e t}$ is equal 0 at the moment, the detector receives the trigger signal of a new pule start. For practical reasons, we perform the integration in the time domain by using the following conversion between wavelength and time. Neutrons produced at the source at time $t_{s r c}$ and arriving at the detector at time $t_{d e t}$ have a wavelength $\lambda$ defined by equation (A2):

$$
\lambda\left(t_{d e t}, t_{s r c}\right)=\frac{\left(t_{d e t}-t_{s r c}\right) h}{m L_{d e t}}=k\left(t_{d e t}-t_{s r c}\right)
$$




$$
\text { with } k=\frac{h}{m L_{d e t}}
$$

where $t_{d e t}-t_{\text {src }}=t_{T O F}$ (see equation (4) and Figure 12).

When integrating in the time domain with the variable $t_{s r c}$ with a fixed value of $t_{d e t}, d \lambda$ can be replaced as follows:

$$
d \lambda=-k \cdot d t_{s r c}
$$

For a single pulse produced by the source the open beam intensity measured at the detector for an arrival time $t_{d e t}$ can be calculated by integrating over the width of the wavelength respective time window of arriving neutrons. The slowest neutron with the maximal wavelength $\lambda_{\max }$ left the pulse source at $t_{s c r}=0$ and the fastest neutron $\lambda_{\text {min }}$ at $t_{s c r}=t_{p u l s e}$ :

$$
\begin{aligned}
I_{0, e f f}\left(t_{d e t}\right) & =-k \int_{t_{\text {pulse }}}^{0} I_{0}\left[k\left(t_{d e t}-t_{s c r}\right)\right] \eta_{d e t}\left[k\left(t_{d e t}-t_{s c r}\right)\right] d t_{s c r} \\
& =k \int_{0}^{t_{p u l s e}} I_{0}\left[k\left(t_{d e t}-t_{s c r}\right)\right] \eta_{d e t}\left[k\left(t_{d e t}-t_{s c r}\right)\right] d t_{s c r}
\end{aligned}
$$

Note that the minus sign was removed by placing the integration limits in ascending order. Similarly, the intensity measured after a container containing water can be calculated as:

$$
I_{e f f}\left(t_{d e t}\right)=k \int_{0}^{t_{\text {pulse }}} I_{0}\left[k\left(t_{d e t}-t_{s c r}\right)\right] \eta_{d e t}\left[k\left(t_{d e t}-t_{s c r}\right)\right] e^{-\sigma_{T}\left[k\left(t_{d e t}-t_{s c r}\right)\right] \delta_{N} h_{S}} d t_{s c r}
$$

where $I_{0}$ represents the wavelength dependent beam intensity after passing through the empty container and $\eta_{\text {det }}$ represents the wavelength dependent detector efficiency.

In the present case of a repetitive pulse train of pulse duration $t_{\text {pulse }}$ and of period $t_{\text {period }}$, with beam overlap only between neighboring pulses, the equations above have to be modified to take into account the preceding and following pulse. We define the integration boundaries as follow:

$$
\begin{aligned}
& t_{\text {start }}=i \cdot t_{\text {period }} \\
& t_{\text {end }}=i \cdot t_{\text {period }}+t_{\text {pulse }}
\end{aligned}
$$


Where $i$ has a value of -1 for the preceding pulse, 0 for the current pulse and 1 for the following pulse. The intensity after the empty container for each $t_{d e t}$ is then calculated as:

$$
I_{0, e f f}\left(t_{\text {det }}\right)=k \sum_{i=-1}^{1}\left[\int_{i \cdot t_{\text {period }}}^{i \cdot t_{\text {period }}+t_{\text {puse }}} I_{0}\left[k\left(t_{\text {det }}-t_{\text {scr }}\right)\right] \eta_{\text {det }}\left[k\left(t_{\text {det }}-t_{\text {scr }}\right)\right] d t_{\text {scr }}\right]
$$

And the intensity after the container filled with water as:

$$
I_{e f f}\left(t_{d e t}\right)=k \sum_{i=-1}^{1}\left[\int_{i \cdot t_{\text {period }}}^{i \cdot t_{\text {period }}+t_{\text {pulse }}} I_{0}\left[k\left(t_{\text {det }}-t_{\text {scr }}\right)\right] \eta_{\text {det }}\left[k\left(t_{\text {det }}-t_{s c r}\right)\right] e^{-\sigma_{T}\left[k\left(t_{d e t}-t_{s c r}\right)\right] \delta_{N} h_{s}} d t_{s c r}\right]
$$

The effective cross section for each $t_{d e t}$ can finally be calculated as:

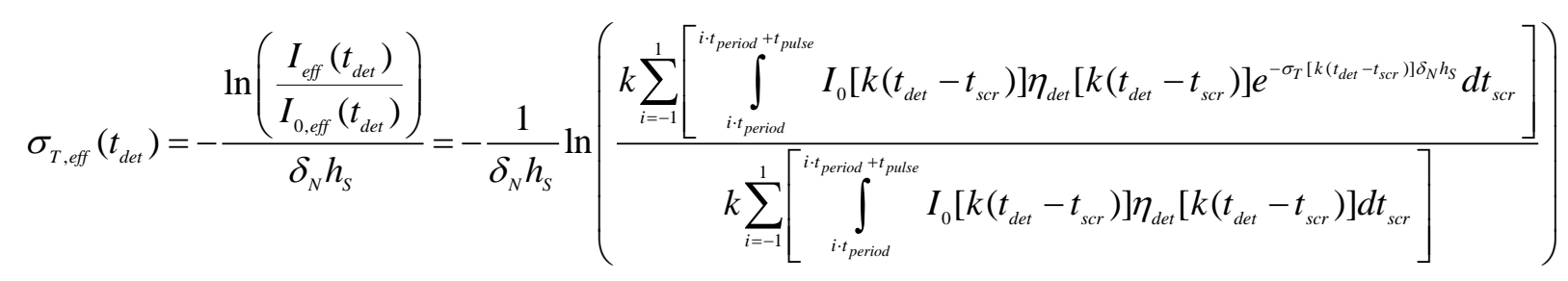

This equation is the one used for the calculation of the effective cross sections presented in Figure 9 and Figure 10. It must be noted that the effective cross section depends on the material thickness and density, a phenomenon known as beam hardening.

Using the same integration boundaries as above for the calculation of $I_{0, \text { eff }}$ for a single pulse we can also calculate the neutron intensity weighted average wavelength (effective wavelength $\lambda_{\text {eff }}$ ) as a function of $t_{d e t}$ :

$$
\lambda_{e f f}\left(t_{d e t}\right)=\frac{\int_{0}^{t_{\text {pulse }}} \lambda\left(t_{\text {det }}, t_{s r c}\right) \cdot I\left[\lambda\left(t_{d e t}, t_{s r c}\right)\right] d t_{s r c}}{\int_{0}^{t_{p u l s e}} I\left[\lambda\left(t_{d e t}, t_{s r c}\right)\right] d t_{s r c}}
$$

\section{Effective cross section for selected time windows}

The effective cross section for a given time window is further given by integrating over a given range of the variable $t_{d e t}$. In this work, a time window for short wavelengths (SW) and one for long wavelengths (LW) was selected. The effective transmissions for these two windows are calculated as: 


$$
\begin{aligned}
& T_{L W}=\frac{\int_{t_{L W_{\min }}}^{t_{L W_{\max }}} I_{e f f}\left(t_{d e t}\right) d t_{d e t}}{\int_{t_{L W_{\min }}}^{t_{\max }} I_{0, e f f}\left(t_{d e t}\right) d t_{d e t}} \\
& T_{S W}=\frac{\int_{t_{S W_{\min }}}^{t_{S W_{\max }}} I_{\text {eff }}\left(t_{d e t}\right) d t_{d e t}}{\int_{t_{S W_{\min }}} I_{0, e f f}\left(t_{d e t}\right) d t_{d e t}} .
\end{aligned}
$$

For small water/ice thicknesses, the exponential expression for the calculation of attenuation coefficients can be linearized using the following approximation:

$$
1-x \cong e^{-x}
$$

Attenuation values for the two wavelength windows are then calculated by applying the linearization approximation to equation (5) and (6):

$$
\begin{gathered}
\Sigma_{L W}=\frac{1-T_{L W}}{h_{S}} \\
\Sigma_{S W}=\frac{1-T_{S W}}{h_{S}}
\end{gathered}
$$

The contrast between the relative attenuation coefficients of super-cooled water and ice is calculated using equation (8). In case the relative attenuation coefficients are estimated using the linearization approximation for small thicknesses (equation (A13)), we can estimate statistical noise (originating from shot noise) based on considerations about their standard deviation. Hayya et al. published a note on the ratio of two normally distributed variables ${ }^{57}$. By reshaping equation 8 of their publication and neglecting the last term because the measurements over the two different intervals can be considered independent, the standard deviation of the ratio $z=\frac{y}{x}=\frac{\Sigma_{L W}}{\Sigma_{S W}}$ can be written as follows:

$$
\sigma_{z}=\frac{\mu_{y}}{\mu_{x}} \sqrt{\left(\frac{\sigma_{x}}{\mu_{x}}\right)^{2}+\left(\frac{\sigma_{y}}{\mu_{y}}\right)^{2}}
$$


The mean or expectation value $\mu_{y}$ is equal to $\Sigma_{L W} h_{S}, \mu_{x}$ is equal to $\Sigma_{s w} h_{s}$ and the standard deviation of $\Sigma h_{S}$ is described by shot noise, which is equal to $\frac{1}{\sqrt{I}}$. Inserting these variables in equation (A16) leads to equation (A17):

$$
\sigma_{\alpha_{r e l}}=\frac{\Sigma_{L W}}{\sum_{S W}}\left(\sqrt{\frac{1}{I_{S W} \Sigma_{S W}^{2}}+\frac{1}{I_{L W} \Sigma_{L W}^{2}}}\right) \frac{1}{h_{S}}
$$

Finally, the contrast-to-noise ratio (CNR) is defined as the ratio of the difference and mean standard deviation of the relative attenuation coefficients of super-cooled water and ice:

$$
C N R=\frac{\alpha_{r e l, s c}-\alpha_{r e l, i c e}}{\frac{1}{2}\left(\sigma_{\alpha_{r e l, s c}}+\sigma_{\alpha_{r e l, i c e}}\right)} .
$$

\section{References}

${ }^{1}$ N. Kardjilov, I. Manke, R. Woracek, A. Hilger and J. Banhart, Materials Today 21, (2018).

${ }^{2}$ P. Boillat, E. H. Lehmann, P. Trtik and M. Cochet, Current Opinion in Electrochemistry 5, (2017).

3J. P. Owejan, T. A. Trabold, J. J. Gagliardo, D. L. Jacobson, R. N. Carter, D. S. Hussey and M. Arif, Journal of Power Sources 171, (2007).

${ }^{4}$ I. Manke, C. Hartnig, M. Grunerbel, J. Kaczerowski, W. Lehnert, N. Kardjilov, A. Hilger, J. Banhart, W. Treimer and M. Strobl, Appl Phys Lett 90, (2007).

${ }^{5}$ A. Lange, A. Kupsch, M. P. Hentschel, I. Manke, N. Kardjilov, T. Arlt and R. Grothausmann, Journal of Power Sources 196, (2011).

${ }^{6}$ P. Boillat, P. Oberholzer, A. Kaestner, R. Siegrist, E. H. Lehmann, G. G. Scherer and A. Wokaun, J Electrochem Soc 159, (2012).

${ }^{7}$ P. Stahl, J. Biesdorf, P. Boillat, J. Kraft and K. A. Friedrich, Journal of The Electrochemical Society 162, (2015).

${ }^{8}$ C. Lee, R. Banerjee, N. Ge, J. K. Lee, B. Z. Zhao, E. Baltic, J. M. LaManna, D. S. Hussey, D. L. Jacobson, R. Abouatallah, R. Wang and A. Bazylak, Electrochim Acta 279, (2018).

${ }^{9}$ O. F. Selamet, U. Pasaogullari, D. Spernjak, D. S. Hussey, D. L. Jacobson and M. D. Mat, Int J Hydrogen Energ 38, (2013).

${ }^{10}$ J. Seweryn, J. Biesdorf, T. J. Schmidt and P. Boillat, Journal of The Electrochemical Society 163, (2016).

${ }^{11}$ R. Hassanein, H. O. Meyer, A. Carminati, M. Estermann, E. Lehmann and P. Vontobel, J Phys D Appl Phys 39, (2006).

${ }^{12}$ P. Zhang, F. H. Wittmann, P. Lura, H. S. Muller, S. B. Han and T. J. Zhao, Cement Concrete Res 108, (2018). 
${ }^{13}$ H. Justnes, K. Bryhn-Ingebrigtsen and G. O. Rosvold, Advances in Cement Research 6, (1994).

${ }^{14}$ P. Trtik, B. Münch, W. J. Weiss, A. Kaestner, I. Jerjen, L. Josic, E. Lehmann and P. Lura, Nuclear Instruments and Methods in Physics Research Section A: Accelerators, Spectrometers, Detectors and Associated Equipment 651, (2011).

${ }^{15}$ P. Benard, E. Kroener, P. Vontobel, A. Kaestner and A. Carminati, Advances in Water Resources 95, (2016).

${ }^{16}$ M. Sedighi-Gilani, M. Griffa, D. Mannes, E. Lehmann, J. Carmeliet and D. Derome, International Journal of Heat and Mass Transfer 55, (2012).

${ }^{17}$ M. Zarebanadkouki, A. Carminati, A. Kaestner, D. Mannes, M. Morgano, S. Peetermans, E. Lehmann and P. Trtik, Physics Procedia 69, (2015).

${ }^{18}$ P. Oberholzer, P. Boillat, R. Siegrist, R. Perego, A. Kästner, E. Lehmann, G. G. Scherer and A. Wokaun, J Electrochem Soc 159, (2011).

${ }^{19}$ A. Santamaria, H. Y. Tang, J. W. Park, G. G. Park and Y. J. Sohn, Int J Hydrogen Energ 37, (2012).

${ }^{20}$ J. Mishler, Y. Wang, P. P. Mukherjee, R. Mukundan and R. L. Borup, Electrochim Acta 65, (2012).

${ }^{21}$ R. Mukundan, J. Davey, R. Lujan, J. Spendelow, Y. S. Kim, D. S. Hussey, D. Jacobson, M. Arif and R. L. Borup, Ecs Transactions 16, (2008).

${ }^{22}$ R. Mukundan, Y. S. Kim, T. Rockward, J. R. Davey, B. Pivovar, D. S. Hussey, D. L. Jacobson, M. Arif and R. Borup, Ecs Transactions 11, (2007).

${ }^{23}$ R. Mukundan, R. Lujan, J. R. Davey, J. S. Spendelow, D. S. Hussey, D. L. Jacobson, M. Arif and R. Borup, Ecs Transactions 25, (2009).

${ }^{24}$ N. Macauley, R. W. Lujan, D. Spernjak, D. S. Hussey, D. L. Jacobson, K. More, R. L. Borup and R. Mukundan, J Electrochem Soc 163, (2016).

${ }^{25}$ Y. Ishikawa, H. Hamada, M. Uehara and M. Shiozawa, Journal of Power Sources 179, (2008).

${ }^{26}$ Y. Ishikawa, M. Shiozawa, M. Kondo and K. Ito, International Journal of Heat and Mass Transfer 74, (2014).

${ }^{27} \mathrm{~N}$. Wakatake, Y. Tabe and T. Chikahisa, Proceedings of ECS Transactions.

${ }^{28}$ B. J. Mason, Adv Phys 7, (1958).

${ }^{29}$ Z. Yang, Q. Du, S. Huo and K. Jiao, Int J Hydrogen Energ 42, (2017).

${ }^{30}$ D. Ko, S. Doh, D. I. Yu, H. S. Park and M. H. Kim, Fuel Cells 18, (2018).

${ }^{31}$ S. H. Ge and C. Y. Wang, Electrochem Solid St 9, (2006).

${ }^{32}$ S. H. Ge and C. Y. Wang, Electrochim Acta 52, (2007).

${ }^{33}$ I. Mayrhuber, F. Marone, M. Stampanoni, T. J. Schmidt and F. N. J. C. Büchi, 2, (2015).

${ }^{34}$ J. Roth, M. Zurbrug, S. Irvine, F. Marone, M. Stampanoni and F. N. Buchi, Ecs Transactions 58, (2013).

${ }^{35}$ J. Biesdorf, P. Oberholzer, F. Bernauer, A. Kaestner, P. Vontobel, E. Lehmann, T. J. Schmidt and P. Boillat, Ecs Transactions 58, (2013). 
${ }^{36}$ J. Biesdorf, P. Oberholzer, F. Bernauer, A. Kaestner, P. Vontobel, E. H. Lehmann, T. J. Schmidt and P. Boillat, Phys Rev Lett 112, (2014).

${ }^{37}$ P. Stahl, J. Biesdorf, P. Boillat and K. A. Friedrich, J Electrochem Soc 163, (2016).

${ }^{38}$ R. Woracek, J. Santisteban, A. Fedrigo and M. Strobl, Nucl Instrum Meth A 878, (2018).

${ }^{39}$ L. Torres, J. R. Granada and J. J. Blostein, Nucl Instrum Meth B 251, (2006).

40J. Biesdorf, ETH-Zürich, 2016.

${ }^{41}$ M. Strobl, M. Bulat and K. Habicht, Nucl Instrum Meth A 705, (2013).

${ }^{42}$ K. Heinloth, Zeitschrift für Physik 163, (1961). EXFOR 21341002

${ }^{43}$ S. B. Stepanov and V. E. Zhitarev, Sov Atom Energy+ 41, (1976). EXFOR 41620002

${ }^{44}$ R. Woracek, T. Hofmann, M. Bulat, M. Sales, K. Habicht, K. Andersen and M. Strobl, Nucl Instrum Meth A 839, (2016).

${ }^{45}$ A. S. Tremsin, V. Dangendorf, K. Tittelmeier, B. Schillinger, M. Schulz, M. Lerche and W. B. Feller, J Instrum 10, (2015).

${ }^{46}$ M. Strobl, Physcs Proc 69, (2015).

${ }^{47}$ A. S. Tremsin, J. V. Vallerga, J. B. McPhate and O. H. W. Siegmund, J Instrum 9, (2014).

${ }^{48}$ P. Boillat, C. Carminati, F. Schmid, C. Grunzweig, J. Hovind, A. Kaestner, D. Mannes, M. Morgano, M. Siegwart, P. Trtik, P. Vontobel and E. H. Lehmann, Opt Express 26, (2018).

${ }^{49}$ C. Carminati, P. Boillat, F. Schmid, P. Vontobel, J. Hovind, M. Morgano, M. Raventos, M. Siegwart, D. Mannes, C. Gruenzweig, P. Trtik, E. Lehmann, M. Strobl and A. Kaestner, Plos One 14, (2019).

${ }^{50}$ A. Furrer, J. Mesot and T. Strassle, Neutron Scattering in Condensed Matter Physics (2009).

${ }^{51}$ R. Macfarlane, D. W. Muir, R. M. Boicourt, I. Kahler, Albert Comstock and J. L. Conlin, (Los Alamos National Lab. (LANL), Los Alamos, NM (United States), 2017).

52J. I. Márquez Damian, J. R. Granada and D. C. Malaspina, Ann Nucl Energy 65, (2014).

53J. I. Márquez Damian, D. C. Malaspina and J. R. Granada, Journal of Chemical Physics 139, (2013).

${ }^{54}$ S. Herdade, M. Bechara, C. Rodriguez and L. Vinhas, (1973). EXFOR 30229003

55J. L. Russell, J. M. Neill and J. R. Brown, (1966). EXFOR 11162003

${ }^{56}$ A. S. Tremsin, J. V. Vallerga, J. B. McPhate and O. H. W. Siegmund, Nucl Instrum Meth A 787, (2015).

${ }^{57}$ J. Hayya, D. Armstrong and N. Gressis, Management Science 21, (1975). 


\section{Figure captions}

Figure 1. Water wedge calibration container, which contained liquid water or ice with thicknesses of $0.13,0.26,0.52$ and $1.01 \mathrm{~mm}$. (a) Top view of container body: the four sections, where the different water/ice thicknesses were accommodated are labeled with 1 - 4 corresponding to Table 1. (b) Cover, (c) closed calibration container.

Figure 2. Transmission images (TI) of the calibration container with ice normalized by TI of dry calibration container. The regions of interests (ROIs) for correction and evaluation areas are depicted as follows: yellow rectangles = scattered background intensity, blue rectangles = beam intensity, black rectangles = evaluation of cross section or $\alpha_{r e l}$. (a) WFM data: 1 - 4 are the 4 water/ice thicknesses corresponding to Table 1 (ROI $4=1.011 \mathrm{~mm}$ water/ice). (b) HDC data: The 30 black squares per section have an area of $1 \mathrm{~mm}^{2}$ and were used to calculate the mean $\alpha_{r e l}$ for the 4 water/ice thicknesses and their standard deviations.

Figure 3. Tls of super-cooled water (a) and ice (b) at $-2{ }^{\circ} \mathrm{C}$. In section 4 , it is clearly visible that the phase transition from super-cooled water to ice resulted in a volume expansion (highlighted with the red rectangle).

Figure 4. Cross section values derived from transmission images measured with WFM choppers for $1.011 \pm 0.01 \mathrm{~mm}$ of ice and super-cooled water at $-2^{\circ} \mathrm{C}$ (dots). Dark error bands show uncertainty due to thickness only and light error band include uncertainty related to correction of scattered background intensity.

Figure 5. (a) Measured cross section (dots) of $1.01 \pm 0.01 \mathrm{~mm}$ of water at $6{ }^{\circ} \mathrm{C}$ (green), super-cooled water at $-2{ }^{\circ} \mathrm{C}$ (red) and ice at $-2^{\circ} \mathrm{C}$ (blue) in comparison to data computed with physical model (solid lines). The range between 2-3 $\AA$ is shown with an insert to make also the small differences in cross section at short wavelengths visible. (b) Computed cross section data for water at $20^{\circ} \mathrm{C}$ (black solid line) and data measured experimentally at room temperature and published in Exfor by Russell and $\mathrm{Neil}^{55}$ (purple rhombus), Heinloth ${ }^{42}$ (orange square), Stepanov et al. ${ }^{43}$ (red stars) and Herdade et al..$^{54}$ (green dots). The measurements of Herdade were scaled by a factor of 1.03 to match the data of Russell, which shows the correct asymptotic free gas value at high neutron energies.

Figure 6: Mean relative attenuation coefficients (equation (7)) measured with HDC for ice and super-cooled water at $-2^{\circ} \mathrm{C}$ for the four different thicknesses. The error bars show the $99 \%$ confidence interval $\left( \pm 2.576 \sigma_{a_{r e l}}\right)$ for the mean relative attenuation coefficients for an evaluation area of $1 \mathrm{~mm}^{2}$.

Figure 7. Confidence interval for $\alpha_{\text {rel }}$ of super-cooled water and ice at $-2^{\circ} \mathrm{C}$ (left $\mathrm{y}$-axis) and $\mathrm{CNR}$ (right $\mathrm{y}$-axis) for a water/ice thickness of $0.13 \mathrm{~mm}$ as a function of evaluation area extrapolated from the measured standard deviation for $1 \mathrm{~mm}^{2}$ evaluation area.

Figure 8. (a) Wavelength spectrum of V20 as a function of neutron arrival time $t_{T O F}$ at the detector measured with medium wavelength resolution (using the ESS source pulse choppers and band choppers preventing pulse overlap) from reference ${ }^{44}$. (b) Effective cross section $\sigma_{\text {eff }}$ (left y-axis) and neutron counts per $\mathrm{mm}^{2}$ and second (right y-axis) as recorded using HDC-TOF. (c) Flight diagram as a function of measurement time at the detector for HDC-TOF (i.e. with low 
wavelength resolution and pulse overlaps). Neutrons of different wavelengths that arrive at the detector at the same time (T1-T3) are depicted schematically with the partially colored spectra in the top row. In addition, the flightpaths from source to detector are highlighted with the corresponding colors in the flight diagram (c). For T3 also the effect of pulse overlap is shown. Violet represent the fastest and red the slowest neutrons. Duty cycle and pulse period are not the same as in the experiment for the sake of a clear illustration of the effect of pulse overlap.

Figure 9. Effective cross section $\sigma_{e f f}$ of ice and super-cooled water at $-2{ }^{\circ} \mathrm{C}$. Comparison between measured (dots) and calculated (solid lines) $\sigma_{\text {eff }}$ as a function of measurement time at the detector and intensity weighted average wavelength (top x-axis). Areas highlighted in grey correspond to the time and wavelength windows used to calculate $\alpha_{\text {rel }}$ values presented in Figure 6 and Table 4 (SW = short wavelength, LW = long wavelength).

Figure 10: Influence of chopper disk duty cycle (DC) and pulse duration on effective cross section (left y-axis) and neutron flux ( $n$ counts, right $y$-axis) evolving over time shown together with the optimal image processing parameters highlighted in grey (short (SW) and long (LW) wavelength integration windows), which should be chosen to maximize CNR. (a) Contrast $=2 \%, D C=60 \%$, (b) Contrast $=6 \%, D C=38 \%$, (c) Contrast $=11 \%$, DC $=20 \%$.

Figure 11: Maximal achievable CNR as a function of target contrast value (b) for optimal chopper disk duty cycle (a) with optimized pulse duration and image processing parameters (integration boundaries for short and long wavelength windows) calculated for a water/ice thickness of $0.1 \mathrm{~mm}$. The presented HDC measurement was performed close to the green point. For the three red points, pulse and image processing parameters are given in Table 5 and $\sigma_{\text {eff }}$ and neutron flux evolving over the TOF cycle are shown in Figure 10.

Figure 12. (a) Wavelength spectrum of V20 as a function of neutron arrival time $t_{T O F}$ at the detector measured with medium wavelength resolution (using the ESS source pulse choppers and band choppers preventing pulse overlap) from reference $^{44}$. (b) Flight diagram illustrating integration boundaries to calculate effective cross section in the time domain for fixed values of measurement time at the detector $t_{d e t}$.

\section{Tables}

Table 1: Measured space between body and cover of water wedge calibration container for the four different sections $(1$ - 4), where water/ice was accommodated during the experiments.

\begin{tabular}{|c|c|c|c|}
\hline Section & $\begin{array}{c}\text { Measured } \\
\text { average } \\
\text { thickness [mm] }\end{array}$ & $\begin{array}{c}\text { Minimal measured } \\
\text { thickness [mm] }\end{array}$ & $\begin{array}{c}\text { Maximal measured } \\
\text { thickness [mm] }\end{array}$ \\
\hline $\mathbf{1}$ & 0.133 & 0.126 & 0.140 \\
\hline $\mathbf{2}$ & 0.261 & 0.251 & 0.272 \\
\hline $\mathbf{3}$ & 0.515 & 0.501 & 0.518 \\
\hline $\mathbf{4}$ & 1.011 & 1.000 & 1.019 \\
\hline
\end{tabular}


Table 2: Chopper settings for WFM and HDC experiment. *Wavelength resolution for HDC is given for intensity weighted average wavelength.

\begin{tabular}{|c|c|c|c|c|c|c|c|c|}
\hline & $\begin{array}{l}\text { Chopper } \\
\text { system }\end{array}$ & $\begin{array}{l}\text { Chopper } \\
\text { settings }\end{array}$ & $\begin{array}{c}\text { Chopper } \\
\text { to } \\
\text { detector } \\
\text { distance } \\
L_{d e t}\end{array}$ & $\begin{array}{l}\text { Rotation } \\
\text { frequency }\end{array}$ & $\begin{array}{c}\text { Pulse } \\
\text { period } \\
t_{\text {period }}\end{array}$ & $\begin{array}{c}\text { Pulse } \\
\text { burst } \\
\text { time } \\
t_{\text {pusse }}\end{array}$ & $\begin{array}{c}\text { Slowest } \\
\text { neutron } \\
\text { arrival } \\
\text { time } \\
\text { after } \\
\text { pulse } \\
\text { start }\end{array}$ & $\begin{array}{c}\text { Wavelength } \\
\text { resolution } \\
\frac{\Delta \lambda}{\lambda}\end{array}$ \\
\hline Unit & - & - & $\mathrm{m}$ & $\mathrm{Hz}$ & $\mathrm{ms}$ & $\mathrm{ms}$ & $\mathrm{ms}$ & $\%$ \\
\hline WFM & $\begin{array}{l}\text { ESS } \\
\text { Source } \\
\text { pulse } \\
\text { and } \\
\text { WFM } \\
\end{array}$ & $\begin{array}{c}\mathrm{Z}_{0}=427 \\
\mathrm{~mm} \\
\text { (maximal) }\end{array}$ & 19.45 & 14 & 71.4 & $\begin{array}{c}<2.8 \text { (ESS } \\
\text { source } \\
\text { chopper) }\end{array}$ & 68.9 & $2 \%$ \\
\hline HDC & $\begin{array}{c}\text { Band } \\
\text { Chopper } \\
1 \text { and } 2 \\
\end{array}$ & $\begin{array}{l}\text { Duty cycle } \\
\quad=30 \%\end{array}$ & 16.3 & 21 & 47.6 & 14.3 & 58.7 & $\begin{array}{c}110 \% \text { at } 2.5 \AA^{*} \\
43 \% \text { at } 8 \AA^{*}\end{array}$ \\
\hline
\end{tabular}

Table 3: Image acquisition settings for WFM and HDC experiment.

\begin{tabular}{|c|c|c|c|c|}
\hline & $\begin{array}{c}\text { Acquisition time per } \\
\text { image stack }\end{array}$ & $\begin{array}{c}\text { Number of } \\
\text { images stacks }\end{array}$ & $\begin{array}{c}\text { Number of time bins (images } \\
\text { in a stack) per TOF cycle }\end{array}$ & $\begin{array}{c}\text { Flux at } \\
\text { detector }\end{array}$ \\
\hline Unit & $\min$ & - & - & $\begin{array}{c}\text { Neutrons / } \\
\text { cm }^{2} \mathbf{s}\end{array}$ \\
\hline WFM & 60 & 5 & 1014 & $2.25^{*} 10^{5}$ \\
\hline HDC & 5 & 2 & 622 & $1.33^{*} 10^{7}$ \\
\hline
\end{tabular}

Table 4: Noise, contrast and CNR for the 4 measured thicknesses.

\begin{tabular}{|c|c|c|c|c|}
\hline Thickness [mm] & $\mathbf{0 . 1 3}$ & $\mathbf{0 . 2 6}$ & $\mathbf{0 . 5 2}$ & $\mathbf{1 . 0 1}$ \\
\hline $\begin{array}{c}\text { Standard deviation of } \\
\text { mean relative attenuation [-] }\end{array}$ & 0.071 & 0.039 & 0.020 & 0.012 \\
\hline Contrast [-] & 7.7 & 8.4 & 8.6 & 8.1 \\
\hline CNR [-] & 1.8 & 3.5 & 6.9 & 10.7 \\
\hline
\end{tabular}


Table 5. Maximal CNR for three target contrast values obtained with optimal duty cycle, pulse period and image processing parameters calculated for a thickness of water/ice of $0.1 \mathrm{~mm}$, an evaluation area of $1 \mathrm{~mm}^{2}$ and 5 minutes image acquisition time.

\begin{tabular}{|c|c|c|c|c|c|c|c|c|c|}
\hline Contrast & $\begin{array}{c}\text { Duty } \\
\text { cycle }\end{array}$ & $\begin{array}{c}\text { Pulse } \\
\text { burst } \\
\text { time }\end{array}$ & Period & $t_{S W_{\min }}$ & $t_{S W_{\max }}$ & $t_{L W_{\min }}$ & $t_{L W_{\max }}$ & $\sigma_{a_{r e l}}$ & CNR \\
\hline$\%$ & $\%$ & $\mathrm{~ms}$ & $\mathrm{~ms}$ & $\mathrm{~ms}$ & $\mathrm{~ms}$ & $\mathrm{~ms}$ & $\mathrm{~ms}$ & - & - \\
\hline 2 & 60 & 30 & 49 & 9 & 39 & 31 & 58 & 0.006 & 4.0 \\
\hline 6 & 38 & 18 & 47 & 7 & 24 & 34 & 52 & 0.012 & 6.7 \\
\hline 11 & 20 & 10 & 47 & 5 & 11 & 40 & 51 & 0.053 & 3.7 \\
\hline
\end{tabular}

Table 6: Variables used for the CNR optimization.

\begin{tabular}{|c|c|c|}
\hline$\alpha_{\text {rel }}$ & - & Relative attenuation coefficient \\
\hline CNR & - & Contrast-to-noise ratio \\
\hline$\delta_{m}$ & $\mathrm{~kg} \mathrm{~cm} \mathrm{~cm}^{-3}$ & Mass density \\
\hline$\delta_{N}$ & Atoms $\mathrm{cm}^{-3}$ & Atomic number density \\
\hline$h$ & $\mathrm{JS}$ & Planck constant \\
\hline$h_{s}$ & $\mathrm{~cm}$ & Thickness of water/ice in water wedge calibration container \\
\hline$i$ & - & Number of pulse $(i=-1,0,1)$ \\
\hline$I_{0}$ & - & Number of neutrons transmitted through dry calibration container \\
\hline$I$ & - & $\begin{array}{l}\text { Number of neutrons transmitted through calibration container } \\
\text { containing water }\end{array}$ \\
\hline$I_{0, \text { eff }}$ & $\mathrm{cm}^{-1}$ & $\begin{array}{l}\text { Number of neutrons effectively transmitted through dry calibration } \\
\text { container when measuring with low wavelength resolution }\end{array}$ \\
\hline$I_{\text {eff }}$ & $\mathrm{cm}^{-1}$ & $\begin{array}{l}\text { Number of neutrons transmitted effectively through container } \\
\text { containing water when measuring with low wavelength resolution }\end{array}$ \\
\hline$L_{\text {det }}$ & $\mathrm{m}$ & Pulse source to detector distance \\
\hline$\lambda$ & $\AA$ & Neutron wavelength \\
\hline$\lambda_{\text {eff }}$ & $\AA$ & $\begin{array}{l}\text { Effective neutron wavelength: Neutron intensity weighted average of } \\
\text { all wavelengths arriving at the same time at the detector }\end{array}$ \\
\hline$m$ & $\mathrm{~kg}$ & Mass of neutron \\
\hline$\eta_{\text {det }}$ & - & Efficiency of detector \\
\hline$\sigma_{a_{r e l}}$ & - & Standard deviation of relative attenuation coefficient \\
\hline$\sigma_{e f f}$ & $\mathrm{~cm}^{-1}$ & $\begin{array}{l}\text { Cross section measured effectively when measuring with low } \\
\text { wavelength resolution }\end{array}$ \\
\hline$\sigma_{T}$ & barn & Total cross section per molecule \\
\hline$\Sigma$ & $\mathrm{cm}^{-1}$ & Attenuation coefficient \\
\hline$\Sigma_{e f f}$ & $\mathrm{~cm}^{-1}$ & $\begin{array}{l}\text { Attenuation coefficient measured effectively when measuring with low } \\
\text { wavelength resolution }\end{array}$ \\
\hline$\Sigma_{L W}$ & - & Attenuation coefficient for chosen long wavelength window \\
\hline$\Sigma_{S W}$ & - & Attenuation coefficient for chosen short wavelength window \\
\hline$t_{d e t}$ & $\mathrm{~ms}$ & $\begin{array}{l}\text { Measurement time: } t_{d e t}=0 \text { when pulse starts (e.g. leading edge of } \\
\text { chopper disk passes neutron exit) }\end{array}$ \\
\hline
\end{tabular}




\begin{tabular}{|l|l|l|}
\hline$t_{L W_{\min }}$ & $\mathrm{ms}$ & Lower time boundary for long wavelength window \\
\hline$t_{L W_{\max }}$ & $\mathrm{ms}$ & Upper time boundary for long wavelength window \\
\hline$t_{\text {period }}$ & $\mathrm{ms}$ & Pulse period (inverse of pulse frequency) \\
\hline$t_{\text {pulse }}$ & $\mathrm{ms}$ & $\begin{array}{l}\text { Pulse burst time (e.g. chopper disk slit open time, which is the product } \\
\text { of duty cycle and pulse period) }\end{array}$ \\
\hline$t_{S W_{\max }}$ & $\mathrm{ms}$ & Upper time boundary for short wavelength window \\
\hline$t_{S W_{\min }}$ & $\mathrm{ms}$ & Lower time boundary for short wavelength window \\
\hline$t_{T O F}$ & $\mathrm{~ms}$ & Flight time of neutron from source to detector \\
\hline$T_{e f f}$ & - & $\begin{array}{l}\text { Transmission of neutrons measured effectively when measuring with } \\
\text { low wavelength resolution }\end{array}$ \\
\hline$T_{L W}$ & - & Transmission for chosen long wavelength window \\
\hline$T_{S W}$ & - & Transmission for chosen short wavelength window \\
\hline$v$ & $\mathrm{~m} \mathrm{~s}^{-1}$ & Velocity of neutron \\
\hline
\end{tabular}


(a)

WFM

(b)

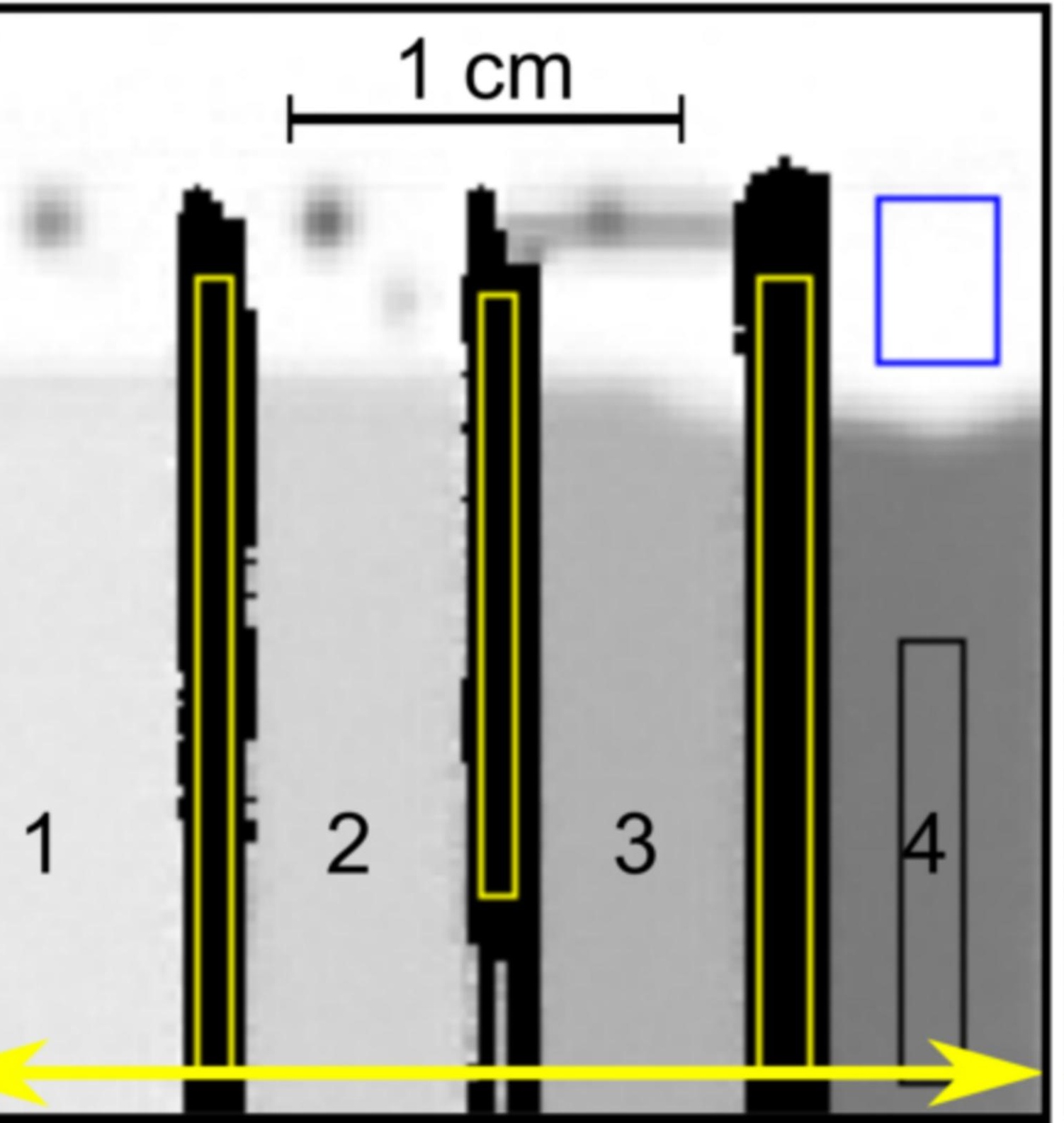

HDC

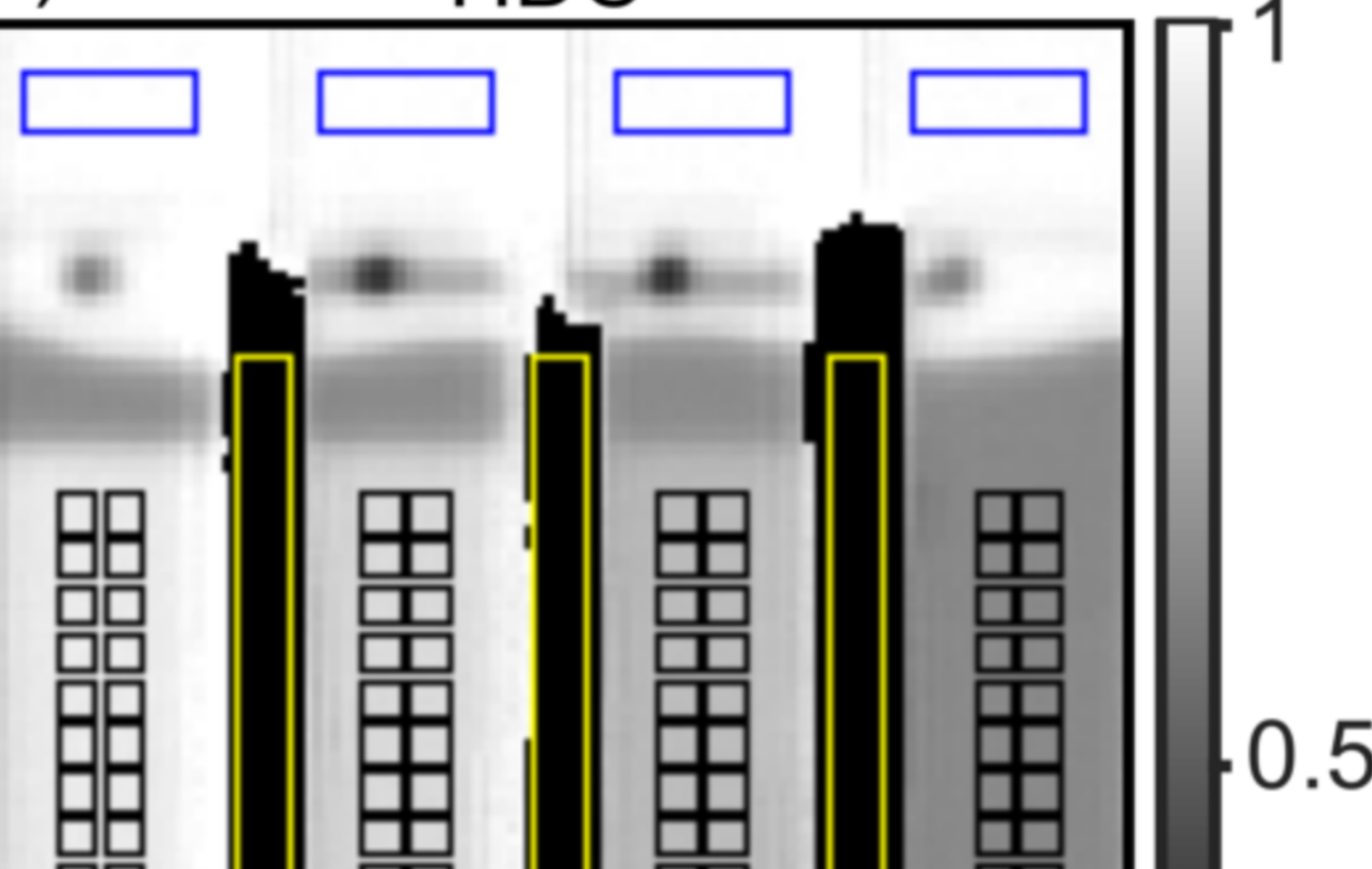


(a)

(b)

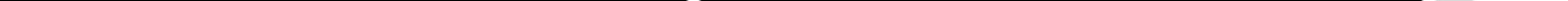




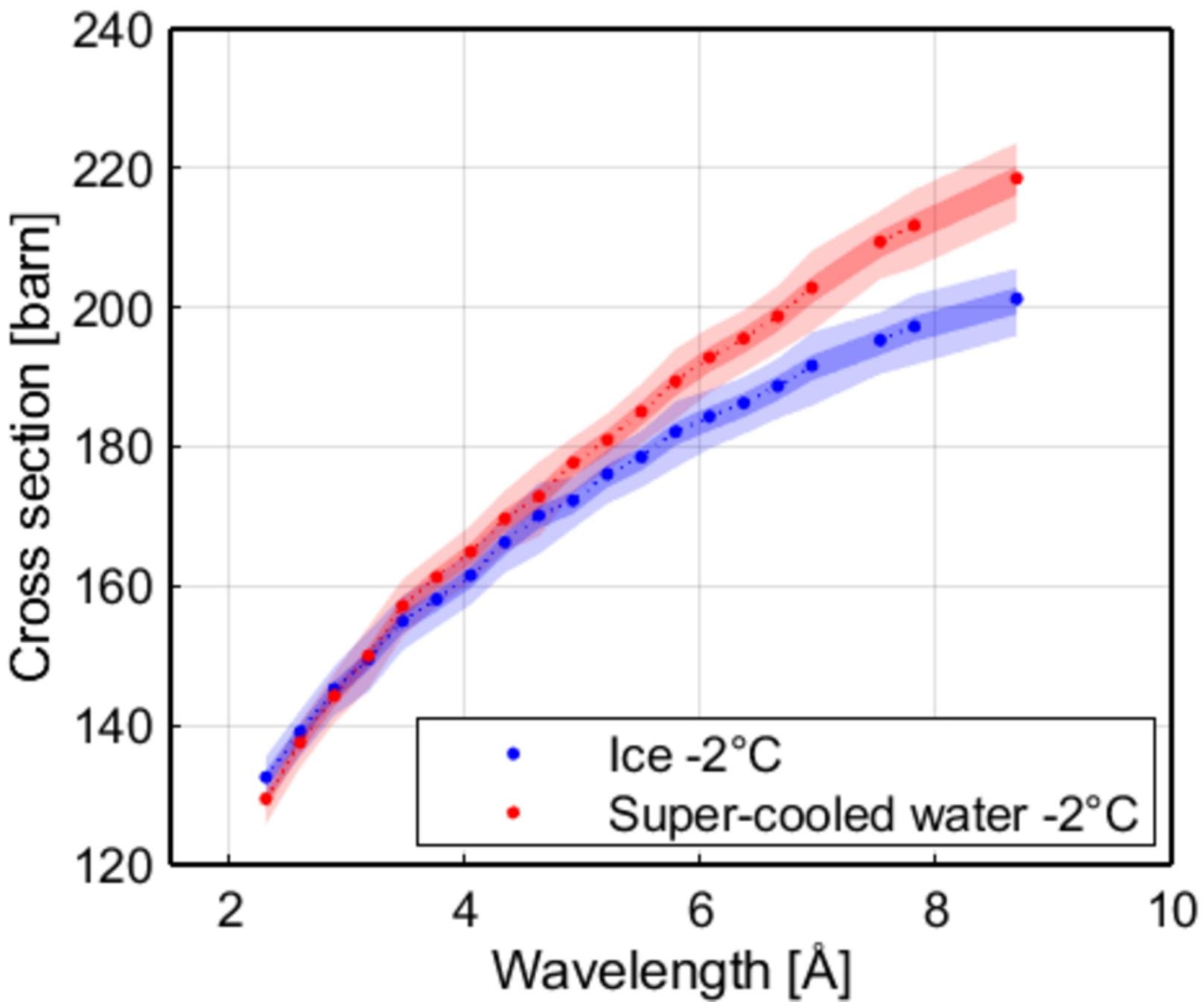




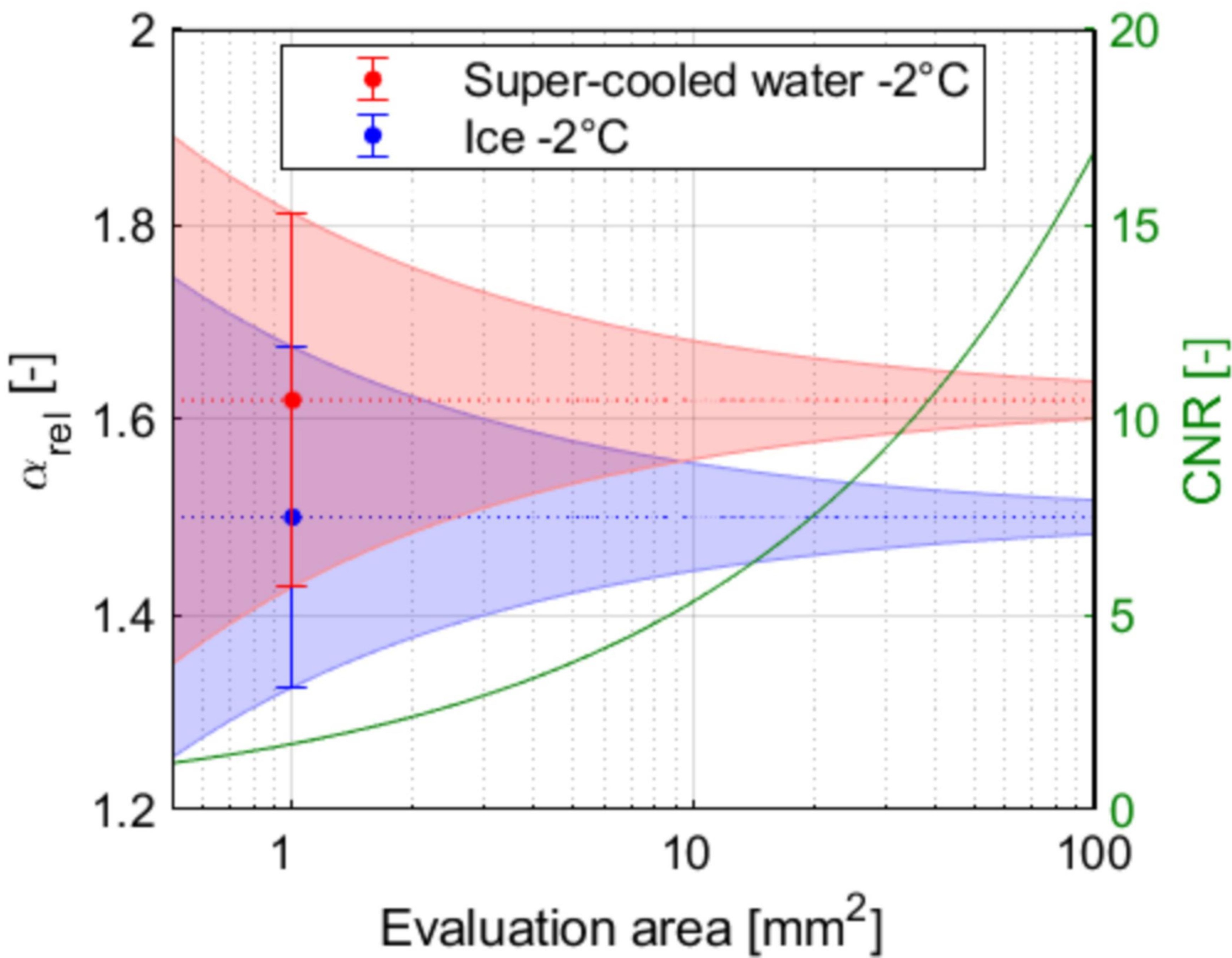


(a)
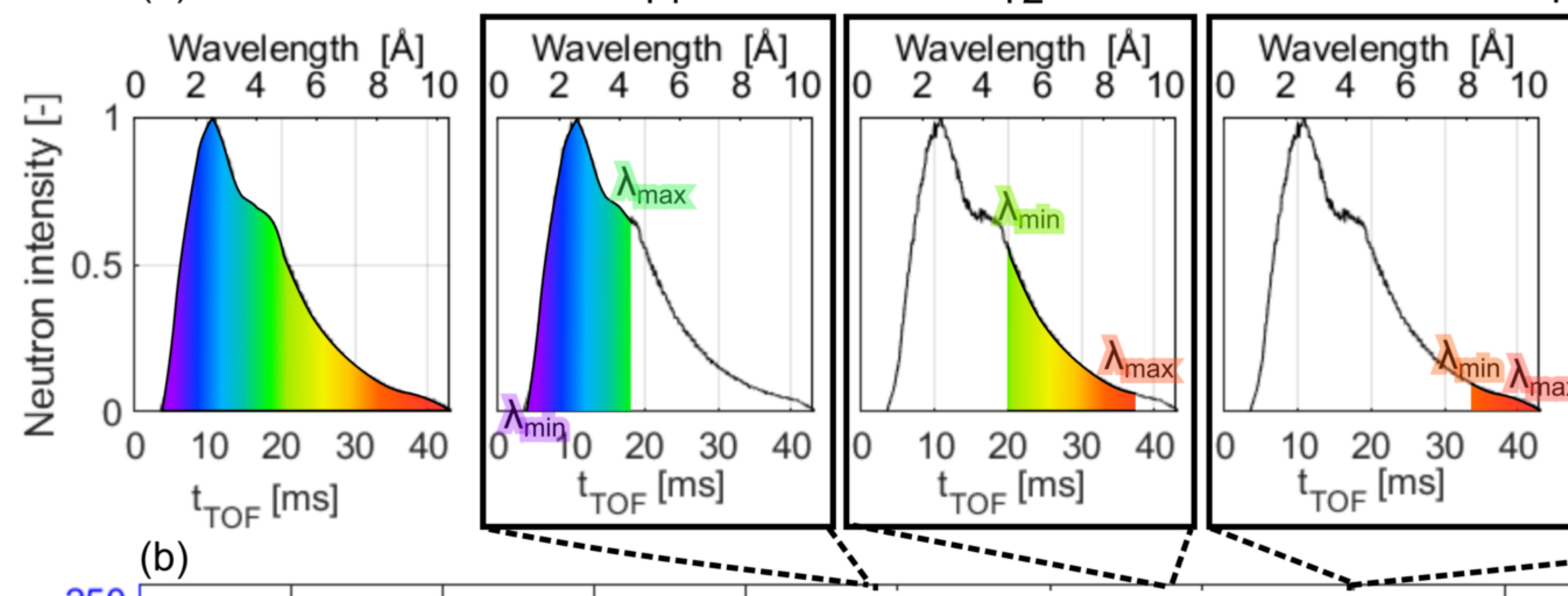

$\begin{array}{lllll}2 & \text { Wavelength } & {[\AA} & \\ 2 & 4 & 6 & 8 & 10\end{array}$

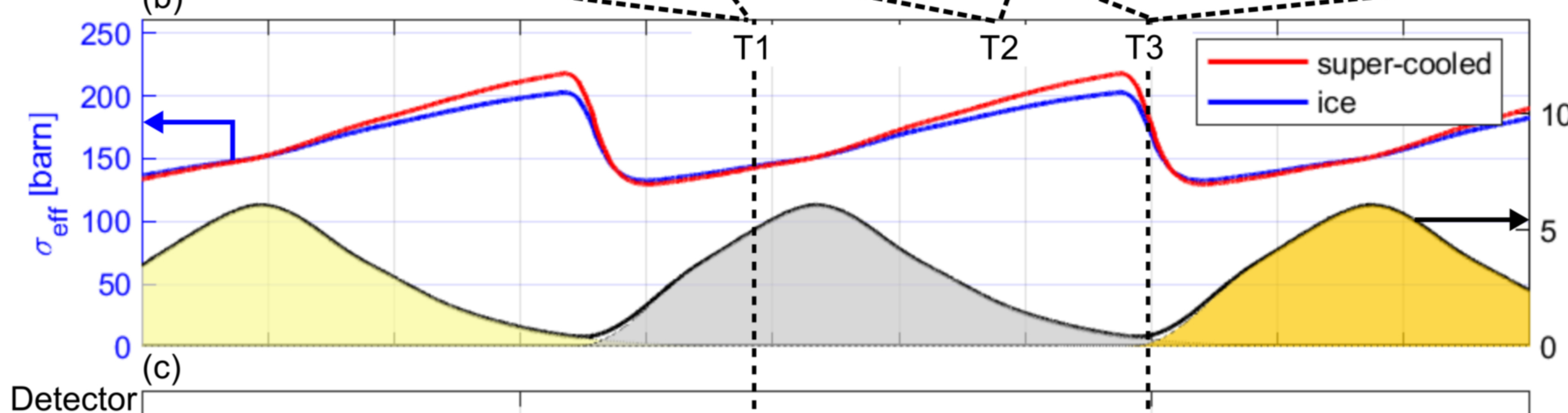

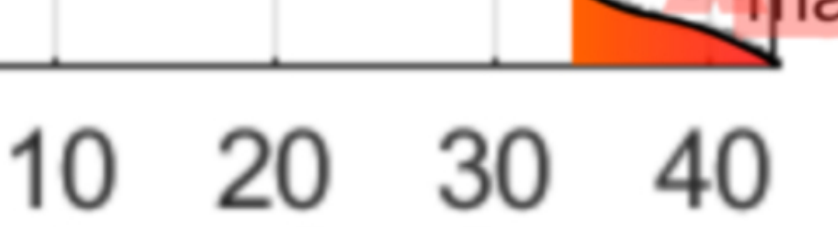

$\mathrm{t}_{\mathrm{TOF}}[\mathrm{ms}]$

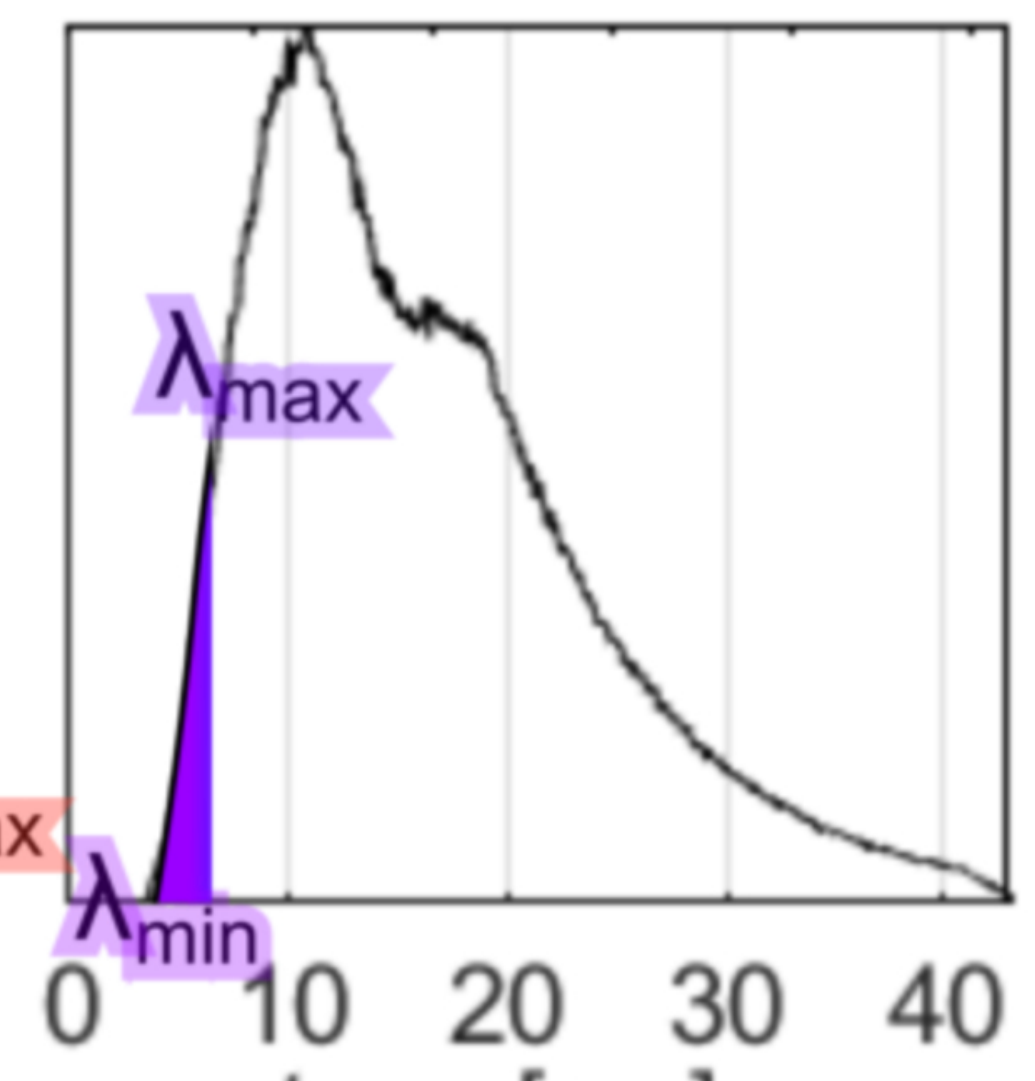

$\mathrm{t}_{\text {TOF }}[\mathrm{ms}]$
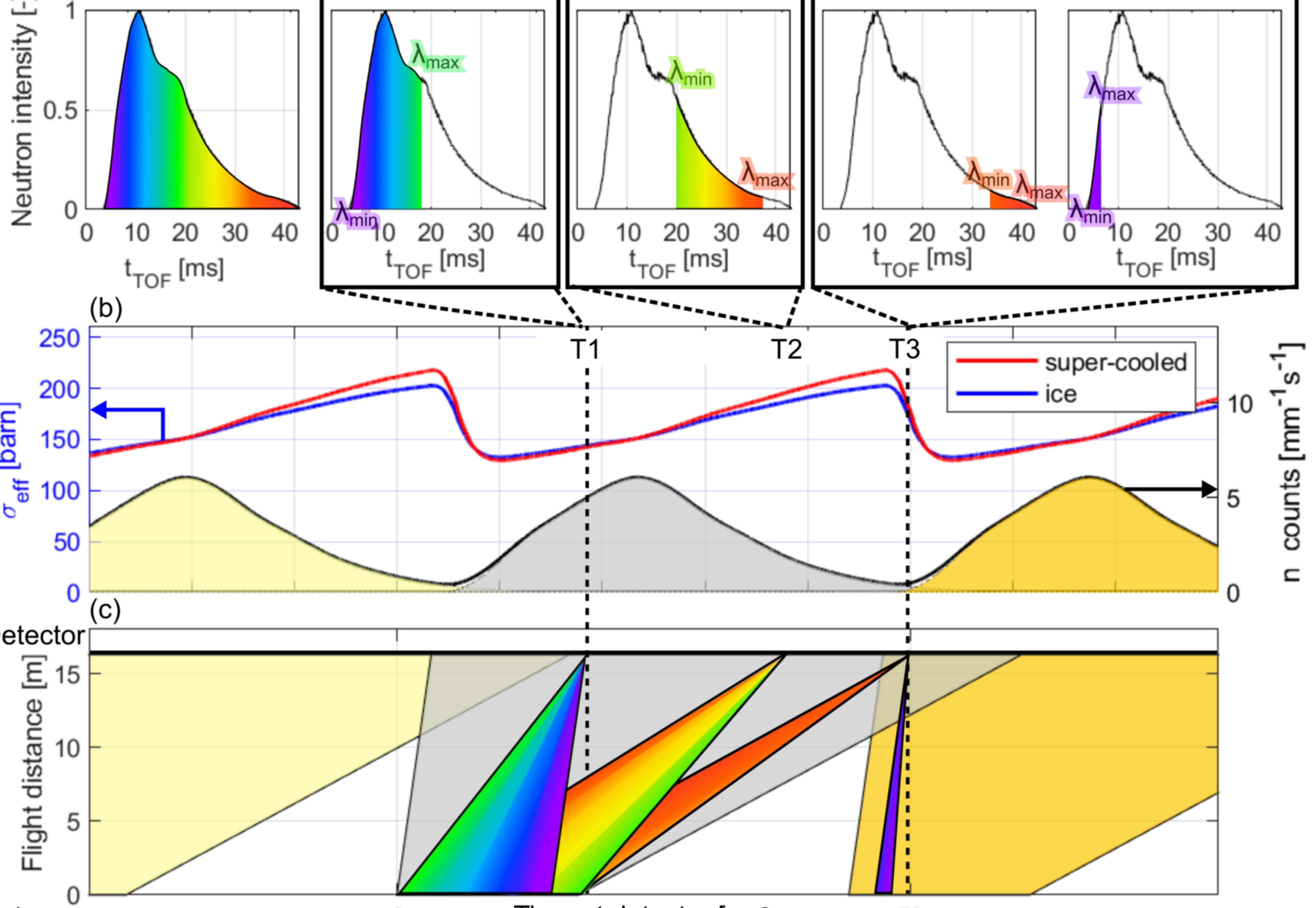

0

50 
Weighted average wavelength $[\AA]$

$\begin{array}{lllllllll}1.9 & 2.5 & 3.1 & 4 & 5.1 & 6.2 & 7.5 & 8.5 & 9.4\end{array}$

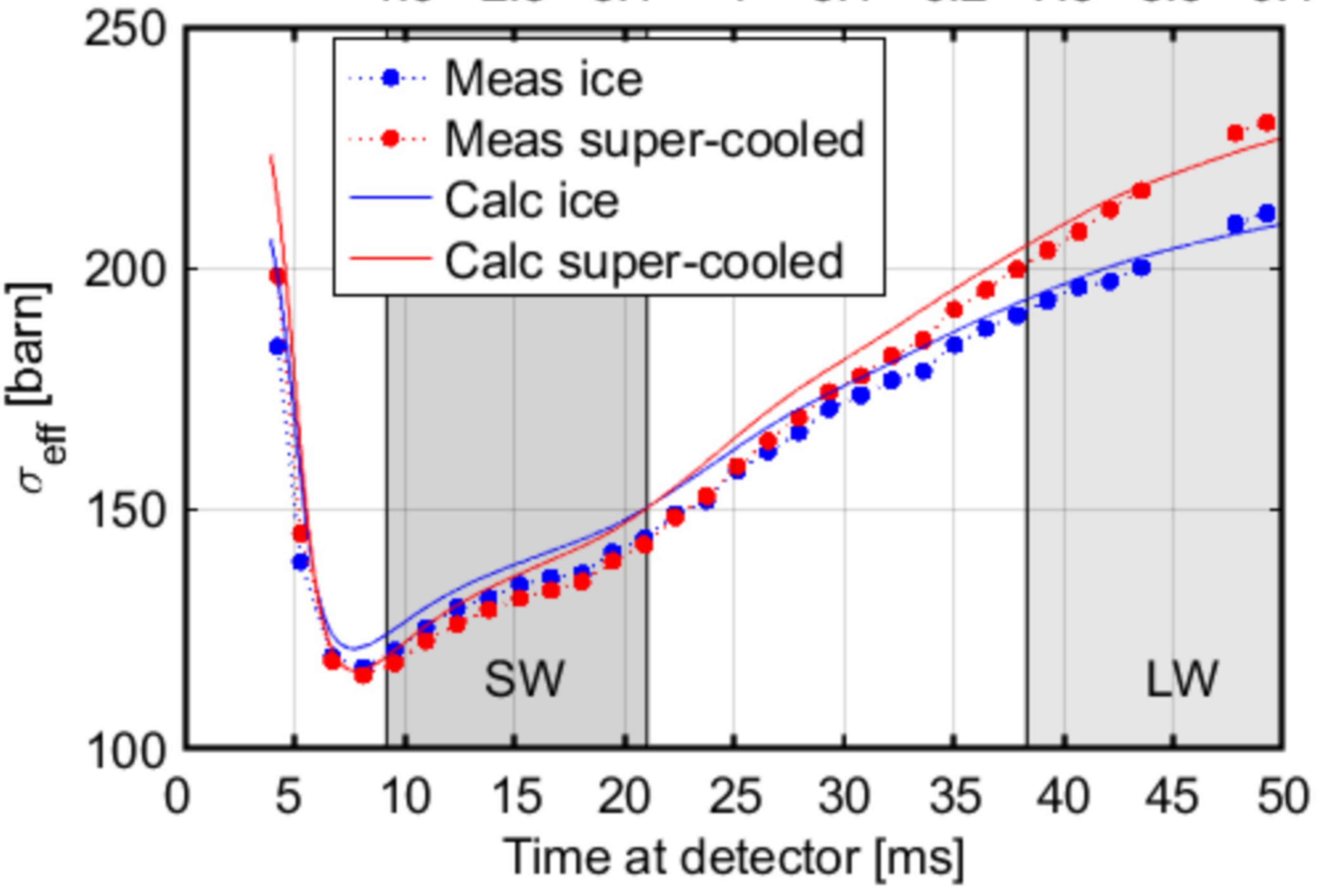



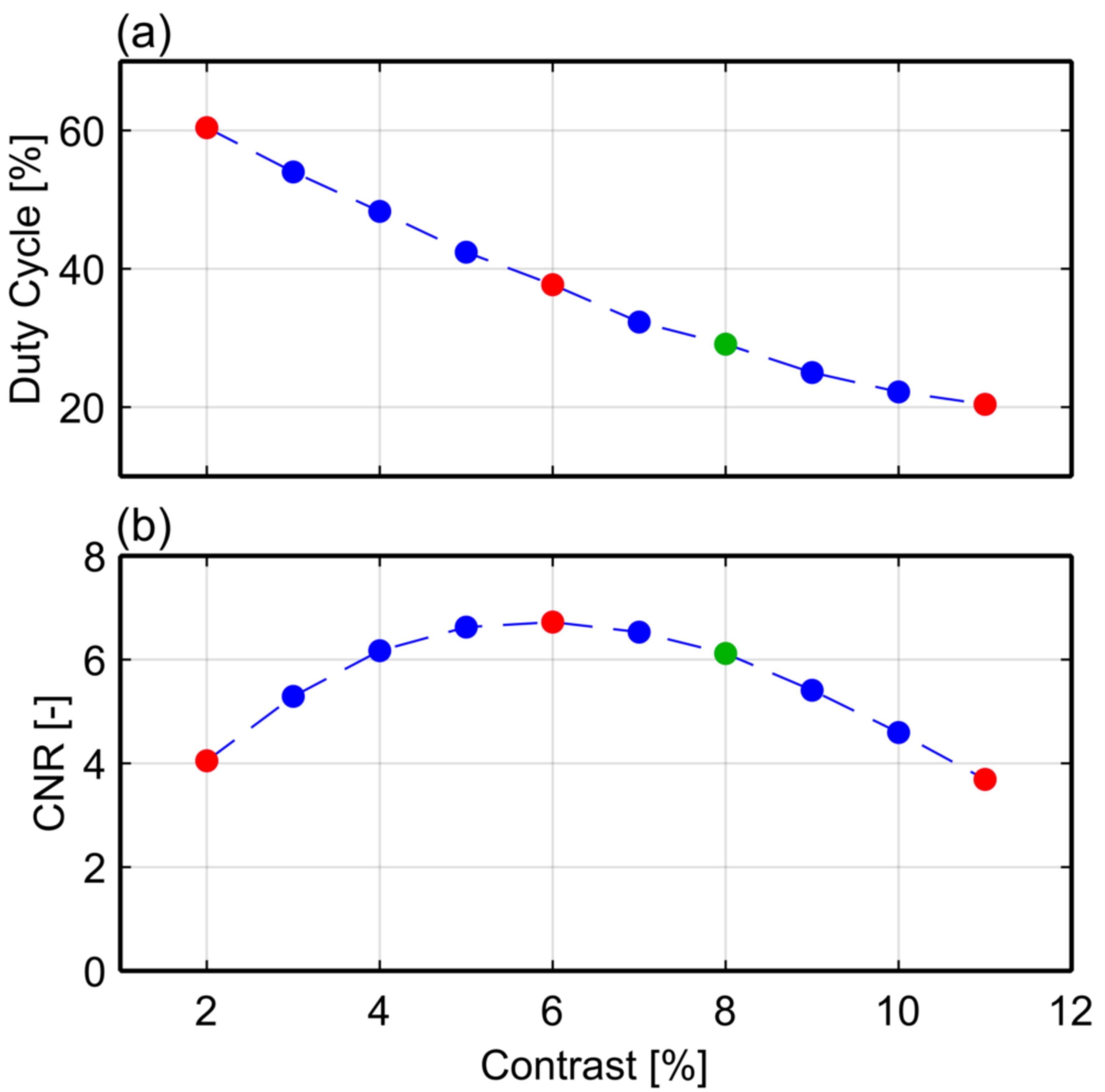
(a)

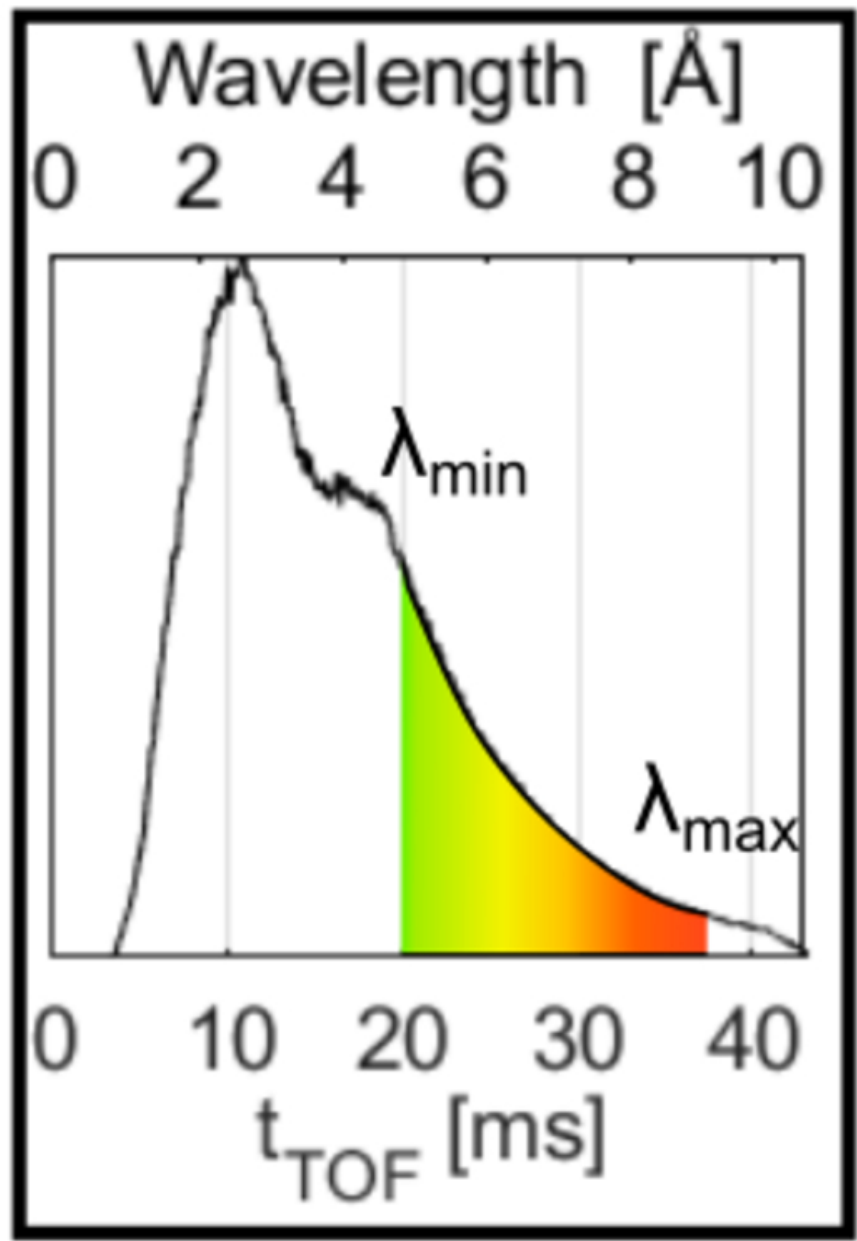

(b)

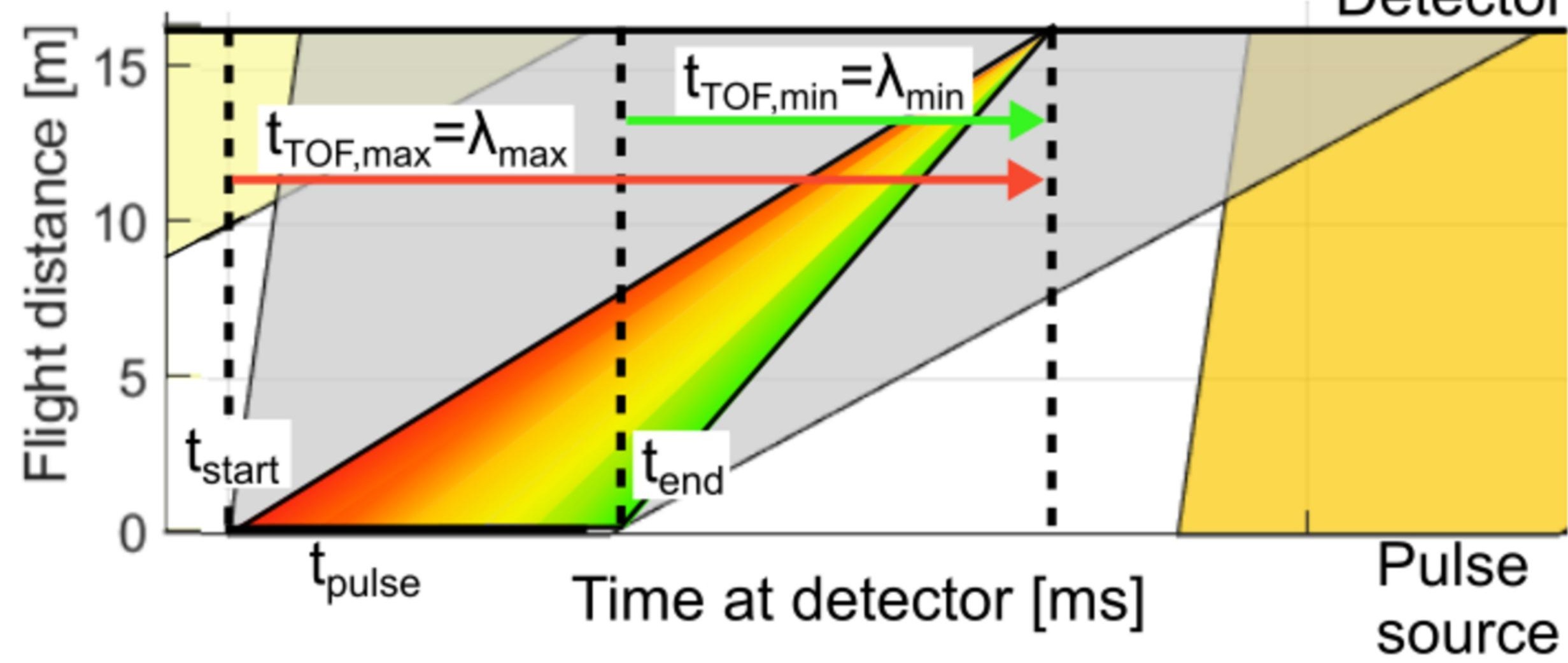

